\title{
Temporal Variability in Thermally Driven Cross-Shore Exchange: The Role of Semidiurnal Tides
}

\author{
HUGO N. ULLOA ${ }^{\mathrm{a}}$ \\ Mechanical and Aerospace Engineering, University of California, San Diego, La Jolla, California
}

KRISTEN A. DAVIS

Civil and Environmental Engineering, University of California, Irvine, Irvine, California

STEPHEN G. MONISMITH

Environmental Fluid Mechanics Laboratory, Stanford University, Stanford, California

GENO PAWLAK

Mechanical and Aerospace Engineering, University of California, San Diego, La Jolla, California

(Manuscript received 6 December 2017, in final form 3 April 2018)

\begin{abstract}
We examine temporal variability of thermally driven baroclinic cross-shore exchange in the context of a tropical fringing reef system focusing on the role of tidally driven alongshore flow. Ensemble diurnal phase averaging of cross-shore flow at the Kilo Nalu Observatory (KNO) in Oahu, Hawaii, shows a robust diurnal signal associated with an unsteady buoyancy/diffusive dynamic balance, although significant variability is observed at subdiurnal time scales. In particular, persistent fortnightly variability in the cross-shore diurnal flow pattern is consistent with modulation by the semidiurnal alongshore tidal flow. The alongshore flow plays a direct role in the cross-shore exchange momentum balance via Coriolis acceleration but also affects the cross-shore circulation indirectly via its influence on vertical turbulent diffusion. An idealized linear theoretical model for thermally driven cross-shore flow is formulated using the long-term time-averaged diurnal dynamic balance at $\mathrm{KNO}$ as a baseline. The model is driven at leading order by the surface heat flux, with contributions from the alongshore flow and cross-shore wind appearing as linear perturbations. Superposition of the idealized solutions for Coriolis and time-varying eddy viscosity perturbations are able to reproduce key aspects of the fortnightly variability. Modifying the model to consider a more realistic alongshore flow and considering effects of nightly convection lead to further improvements in comparisons with KNO observations. The ability of the theoretical approach to reproduce the fortnightly patterns indicates that semidiurnal variations in the alongshore flow are effective in modulating the cross-shore flow via Coriolis and vertical turbulent transport mechanisms.
\end{abstract}

\section{Introduction}

Cross-shore momentum exchange in coastal regions is a fundamental physical process that modulates the mass and heat transport between the nearshore and the adjoining ocean and can regulate biological processes within ecosystems such as coral reefs (Monismith 2007;

\footnotetext{
${ }^{\text {a }}$ Current affiliation: Physics of Aquatic Systems Laboratory, École Polytechnique Fédérale de Lausanne, Lausanne, Switzerland.
}

Corresponding author: Geno Pawlak,pawlak@ucsd.edu
Lowe and Falter 2015). This process can be driven by a variety of mechanisms, including Ekman transport, cross-shelf wind-driven circulation (Lentz and Fewings 2012; Brink 2016), wave-driven circulation (Lowe et al. 2009), internal waves and tides (Leichter et al. 1996; Davis et al. 2008; Davis and Monismith 2011), and buoyancy-driven flows (Monismith et al. 2006; Biton et al. 2008; Molina et al. 2014), all of which have different spatial and temporal scales. The role of these various mechanisms will depend on local characteristics such as bathymetry, shelf width, stratification, and so forth. In particular, field studies have shown that diurnal 


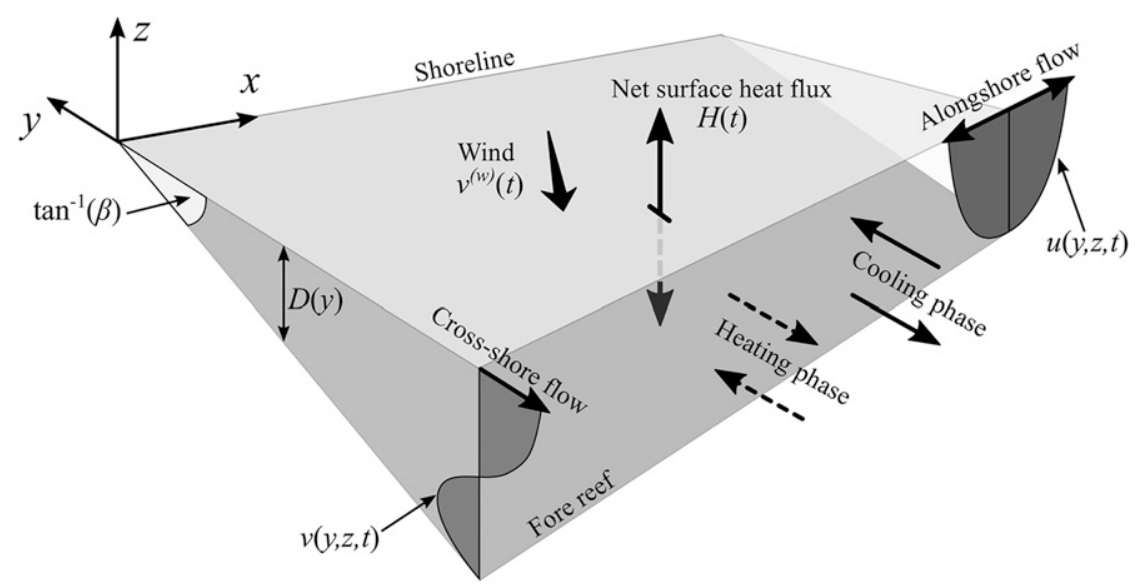

FIG. 1. Schematic of the idealized forereef system. The coordinate system $(x, y, z)$ defines the alongshore, cross-shore, and vertical position, respectively, of a fluid parcel.

surface heat flux is an important and persistent mechanism in driving cross-shore temperature gradients and, as a consequence, thermally driven cross-shore flows at diurnal time scales, particularly in tropical and Mediterranean regions where surface heat fluxes can be high (Monismith et al. 1990, 2006; Molina et al. 2014).

The diurnal signal of the surface heat flux is characterized by a cooling and a heating phase, which causes shallower waters to cool and heat more rapidly than deeper waters, respectively, thus setting up a cross-shore thermal gradient that drives cross-shore baroclinic flows between the nearshore and offshore waters. The circulation pattern associated with the cooling/heating diurnal phase is shown in schematic form in Fig. 1. The fluid response for the cooling phase leads to colder offshore flow at the bottom, balanced by warmer onshore flow at the surface, with a reverse flow pattern for the heating phase (Farrow and Patterson 1993). The phasing of the flow response relative to the diurnal surface heat flux forcing will depend on the dynamic regimes for the buoyancy and cross-shore momentum balances (Farrow and Patterson 1993; Monismith et al. 2006). Although the various mechanisms described above can provide intermittent perturbations to the thermally driven crossshore exchange, persistent forcing mechanisms, such as diurnal winds (Farrow 2013; Lin 2015) and tidally driven alongshore currents, can lead to systematic variability in the regular cross-shore exchange pattern.

Long-term observations of currents and thermal structure at the Kilo Nalu Observatory (KNO) on the south shore of Oahu, Hawaii, show that thermally driven baroclinic exchange is a dominant mechanism for crossshore transport for this tropical forereef environment (Molina et al. 2014). The cross-shore flow is primarily determined by the diurnal surface heat flux and the cross-shore wind stress. Figure 2 highlights the diurnal variation (vertical axis) of the primary forcing mechanisms identified at KNO, from January to September 2010. The net surface heat flux $H$ (Figs. 2a,b) shows a robust diurnal pattern, characterized by regular cooling and heating phases; minor variations in magnitude are evident comparing winter and summer seasons. The cross-shore wind component $v^{(w)}$ (Figs. 2c,d) shows a persistent offshore diurnal pattern, with a minimum in the early morning and a maximum during the afternoon (Fig. 2d). A few exceptional events associated with onshore wind bursts, known as Kona events, are observed over the period shown (positive winds in Fig. 1c). The depth-averaged alongshore flow $\langle u\rangle_{D}$ shows a strong tidal modulation, dominated by semidiurnal constituents (Figs. 2e,f). It is important to note that the alongshore flow has a dominantly barotropic vertical profile (Molina et al. 2014). Detailed information of the study site, instrument deployment, and data processing is found in Pawlak et al. (2009) and Molina et al. (2014).

The strength of the thermal exchange can be quantified in terms of the layer cross-shore exchange velocity,

$$
\left\langle v_{\mathrm{ex}}\right\rangle_{\mathcal{L}}=\frac{1}{\mathcal{L}} \int_{\mathcal{L}} v_{\mathrm{ex}}(t, z) d z
$$

where

$$
v_{\mathrm{ex}}(t, z)=\boldsymbol{v}-\langle\boldsymbol{v}\rangle_{D}-\left(\overline{\boldsymbol{v}}-\langle\overline{\boldsymbol{v}}\rangle_{D}\right)
$$

is the cross-shore baroclinic velocity component, $\mathcal{L}$ represents either the upper- or lower-layer thickness, and $v$ is the observed cross-shore velocity profile. The second term on the RHS in Eq. (2), $\langle v\rangle_{D}$, is the depthaveraged cross-shore velocity (where $D$ is the total depth), which represents the time-varying barotropic 
(a)
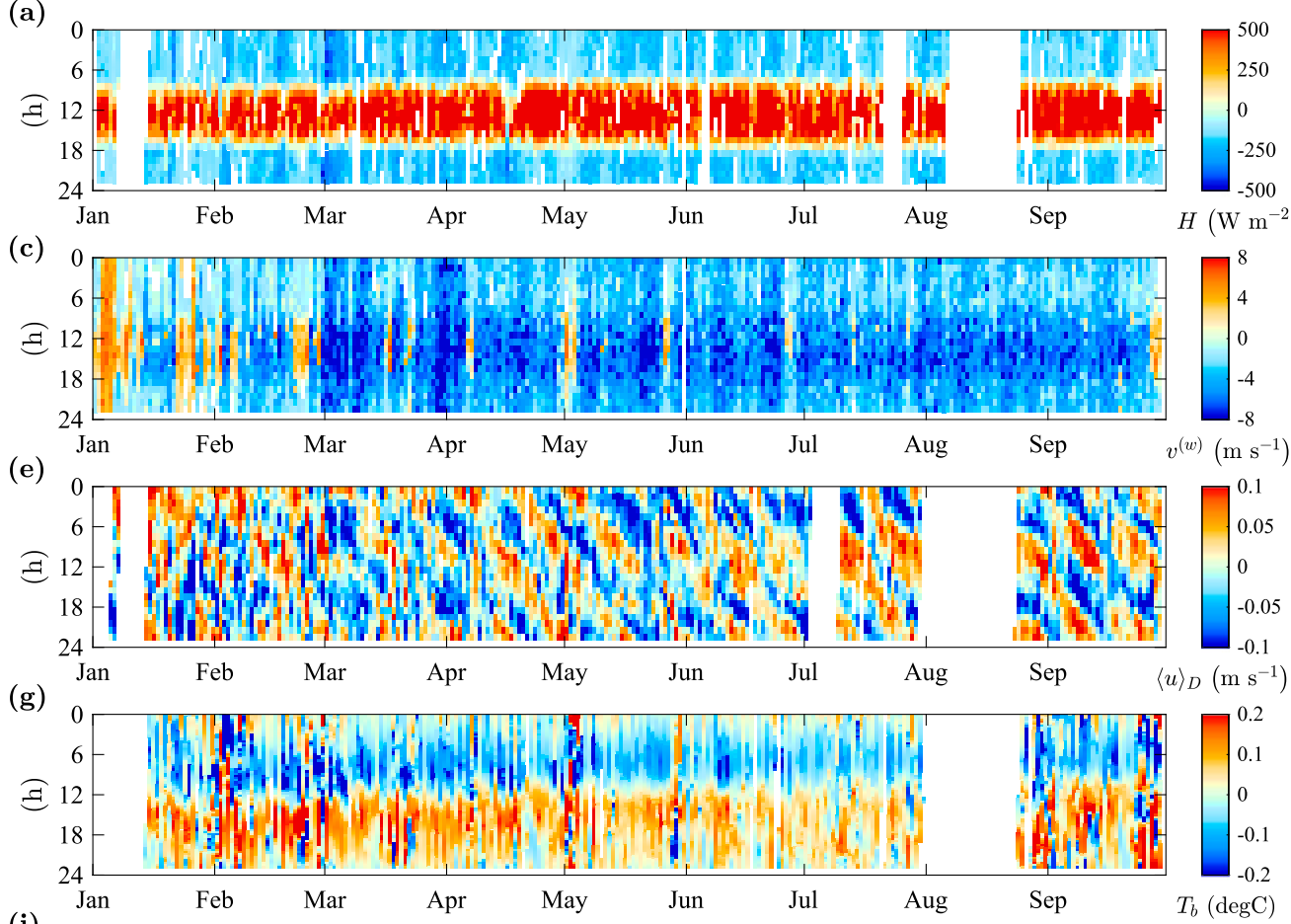

(i)

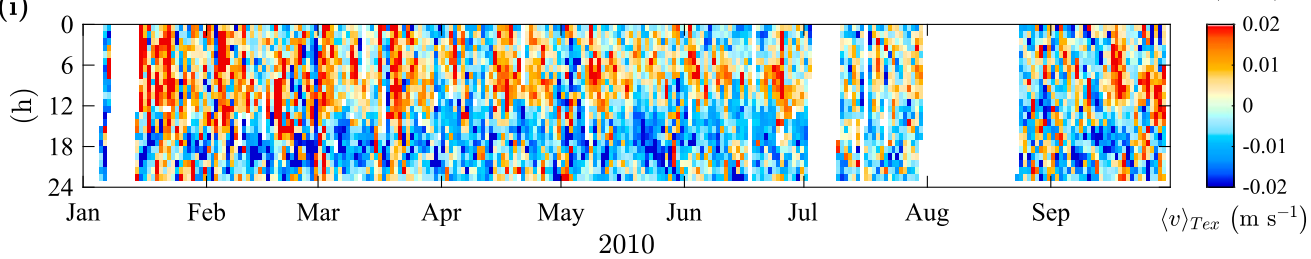

(b)

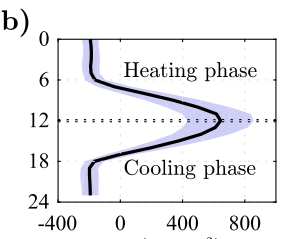

(d)

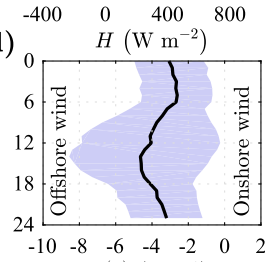

(f)

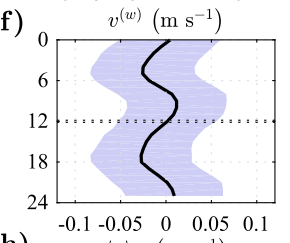

(h)

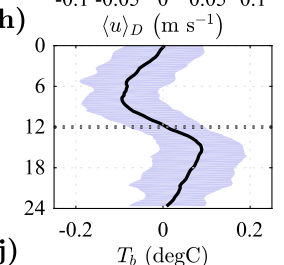

(j)

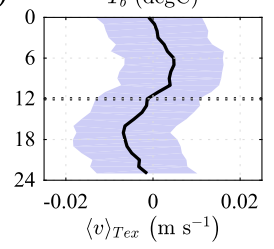

FIG. 2. Observations at KNO for 2010. Diurnal variation of (a) surface heat flux $H$, (c) cross-shore wind component $v^{(w)}$, and (e) depthaveraged alongshore flow $\langle u\rangle_{D}$. Time-averaged diurnal profile of (b) $H$, (d) $v^{(w)}$, and (f) $\langle u\rangle_{D}$. Shading indicates one standard deviation in variations. (g),(h) High-pass-filtered $(33 \mathrm{~h})$ bottom water temperature, along with its time-averaged diurnal profile. (i),(j) Top-layer baroclinic cross-shore velocity $\langle v\rangle_{\mathrm{Tex}}$, along with its time-averaged diurnal profile. Adapted from Molina et al. (2014).

velocity component. The third term on the RHS in Eq. (2), $\left(\bar{v}-\langle\bar{v}\rangle_{D}\right)$, captures the time-averaged baroclinic profile, where $\bar{v}$ is the time-averaged velocity profile and $\langle\overline{\boldsymbol{v}}\rangle_{D}$ is the depth-averaged/time-averaged velocity. The exchange velocity defined in Eq. (2) allows us to isolate the temporal variability in the baroclinic exchange.

Figure 3 shows $v_{\text {ex }}$ versus depth, ensemble averaged by time of day using observations at KNO between January 2007 and September 2011. This represents the most complete and reliable stretch of KNO data. Because the ADCP does not resolve the near-surface flow, we exclude the upper bins ( $\sim 12 \%$ of water column) from the analysis. The flow exhibits a distinctly baroclinic pattern, with surface onshore flow and bottom offshore flow in morning hours $(0-12 \mathrm{~h})$, reversing direction after noon $(12-24 \mathrm{~h})$. The depth at which $v_{\text {ex }}$ changes sign (dotted line) can vary $\sim \pm 1 \mathrm{~m}$ with respect to the mean reversal depth (dashed line), $z \approx-5.5 \mathrm{~m}$. We thus define time-dependent top- and bottom-layer thicknesses based on this variable reversal depth using the long-term flow pattern in Fig. 3, to examine and quantify the temporal variability for the cross-shore exchange.

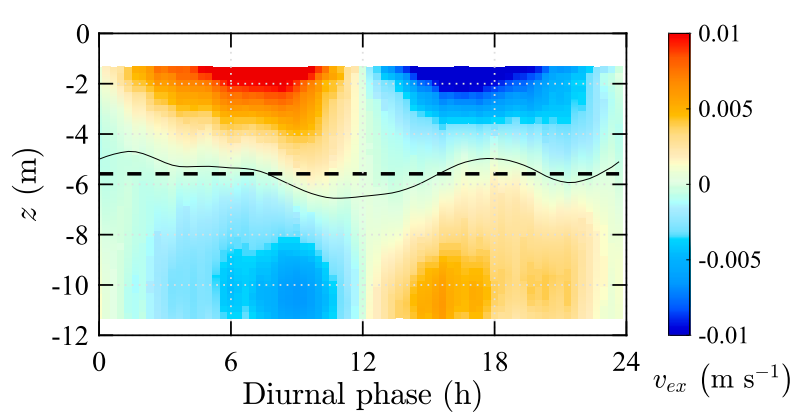

FIG. 3. Ensemble-averaged cross-shore baroclinic velocity component $v_{\text {ex }}(t, z)$, over the period January 2007 to September 2011 vs depth and time of day. 
The top-layer cross-shore exchange velocity, defined using Eq. (2), with $\mathcal{L}$ defined using the diurnally varying reversal depth in Fig. 3, is shown in Figs. 2i,j. The crossshore flow pattern has a marked diurnal profile (Fig. 2j), with a diurnal phase that is well characterized by the dynamic regimes identified by Molina et al. (2014): buoyancy is dominated by an unsteady response to the surface heat flux, while the momentum balance is largely between the baroclinic pressure gradient and vertical turbulent diffusion. This dynamic balance leads to a cross-shore baroclinic pressure gradient and cross-shore velocity that are in quadrature with the surface heat flux. Despite the clear diurnal structure evident in Fig. 2j, however, significant temporal variability is evident over a range of time scales.

Tidal processes, which for KNO are dominated by the $\mathrm{M}_{2}$ frequency $\left(T_{\mathrm{M}_{2}}=12.42 \mathrm{~h}\right)$, can provide a potential source for variability in the cross-shore flow on a fortnightly time scale. In particular, the predominantly tidal alongshore flow can affect the cross-shore exchange directly via the Coriolis exchange contribution (Molina et al. 2014). Additionally, alongshore currents provide a substantial source for turbulence that can control the effective diffusivity, and, hence, the vertical momentum flux. We argue that tidally driven alongshore currents can thus be a persistent source of temporal variability for the cross-shore exchange at KNO and similar open systems at $\mathbf{M}_{2}$ and fortnightly time scales.

Here, we investigate the role of the $\mathrm{M}_{2}$ tidal phase in generating temporal variability in the thermally driven cross-shore exchange at KNO. In section 2 we analyze the cross-shore exchange at KNO via ensemble averages of key measures of the cross-shore momentum transport. In section 3 we formulate a theoretical framework to study the cross-shore exchange as a function of the diurnal and $\mathrm{M}_{2}$ phase. For this, we consider an idealized three-dimensional wedge geometry, with a cross-shore slope $\beta$, to characterize a fringing reef environment (Fig. 1). The system is forced by a periodic heat flux $H(t)$ that acts uniformly over the water column. The crossshore momentum balance is perturbed by including an $\mathrm{M}_{2}$ tidally driven alongshore flow and a diurnally varying cross-shore surface wind stress. The prescribed alongshore flow $u$ affects the cross-shore momentum balance through the Coriolis acceleration and indirectly via its contributions to the turbulent diffusivity $\nu_{t}$. Results and comparison between theory and observations are presented in section 4 . In section 5 we discuss the implications of our main results and the applicability of the theoretical model to other forcing conditions and coastal systems.

\section{Lunar-solar phase variability (observations)}

Two time scales characterize the primary forcing mechanisms for cross-shore exchange, the diurnal solar period, $\mathcal{T}_{S}=24 \mathrm{~h}$, for surface heat flux and the wind, and the lunar $\mathrm{M}_{2}$ tidal period, for alongshore currents. Therefore, we examine the forcing mechanisms and the flow variability in a lunar-solar $\left(\mathrm{M}_{2} /\right.$ diurnal $)$ phase plane $\left(\mathrm{LSP}^{2}\right)$. Ensemble averages for the tidal height, the horizontal depth-averaged currents, and the exchange quantities were calculated from 20-min averages as a function of $\mathrm{M}_{2} /$ diurnal phase, using almost five years of observations (January 2007-September 2011) at KNO. The $\mathrm{M}_{2}$ phase is resolved in 14 bins ( $~ 1$ bin per day) and the diurnal phase is resolved into 24 bins (1-h ensembles). The alongshore direction is defined based on the principal axes for the depth-averaged tidal flow at KNO.

Figures $4 \mathrm{a}, 4 \mathrm{~b}, 4 \mathrm{c}$, and $4 \mathrm{~d}$ show the tidal height $\eta$, the depth-averaged alongshore velocity $\langle u\rangle_{D}$, and the depthaveraged cross-shore velocity $\langle v\rangle_{D}$, along with the number of data points contributing to each ensemble in the $\mathrm{LSP}^{2}$, respectively. The horizontal axis for the $\mathrm{LSP}^{2}$ shows the diurnal phase ( $24 \mathrm{~h}$; where 0000 is midnight). The vertical axis shows the $\mathrm{M}_{2}$ phase corresponding to 0000 for a given day. This phase decreases every day from $\theta / 2 \pi=1$ to 0 over a 14.49 -day cycle. Although the $\mathrm{M}_{2}$ cycle takes 28.98 days to complete, because there are two $M_{2}$ peaks per day the $M_{2}$ phase at midnight is repeated every 14.49 days. Figures $4 \mathrm{a}$ and $4 \mathrm{~b}$ reflect the phase relationship between $\eta$ and $\langle u\rangle_{D}$ but also highlight the diurnal shift of the tidally driven alongshore flow in terms of the fortnightly $\mathrm{M}_{2}$ cycle. The alongshore flow observations thus establish the temporal structure of the Coriolis force in the cross-shore momentum balance.

The magnitude of the cross-shore barotropic component $\langle\boldsymbol{v}\rangle_{D}$ (Fig. 4c) is roughly one order smaller than $\langle u\rangle_{D}$. Despite the weak magnitude, some $\mathbf{M}_{2}$-phase modulation is evident in $\langle v\rangle_{D}$, indicative of a cross-shore tidal response, although the relationship with the alongshore flow is not immediately clear. The depthaveraged cross-shore flow has a mean onshore component, with a significant diurnal variation, as noted by Molina et al. (2014). The diurnal variation accounts for roughly $30 \%$ of the $\mathrm{LSP}^{2}$ variance, with a peak in the early afternoon and a minimum near dawn. This phasing suggests a link with the cross-shore wind (Fig. 2d), which also peaks in the early afternoon, though the wind stress is predominantly offshore. The local onshore transport, which must be balanced by offshore flow elsewhere, is thus likely associated with alongshore variations in the cross-shore wind stress. The remaining variability in $\langle\boldsymbol{v}\rangle_{D}$ is predominantly at the $\mathrm{M}_{2}$ frequency, nearly in quadrature with the alongshore velocity, with offshore 


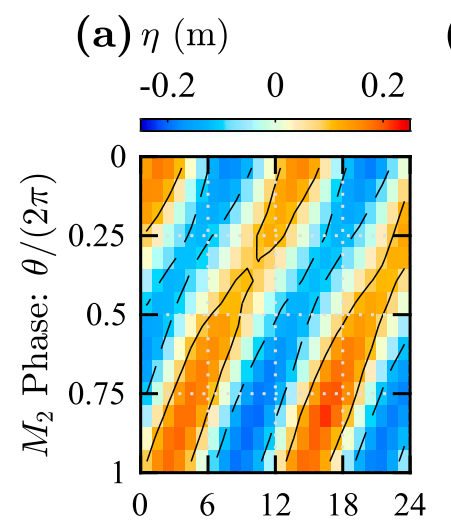

(h) (b) $\langle u\rangle_{D}\left(\mathrm{~m} \mathrm{~s}^{-1}\right)$

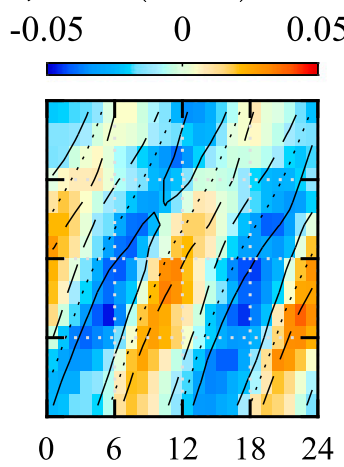

(h) (c) $\langle v\rangle_{D}\left(\mathrm{~m} \mathrm{~s}^{-1}\right)$

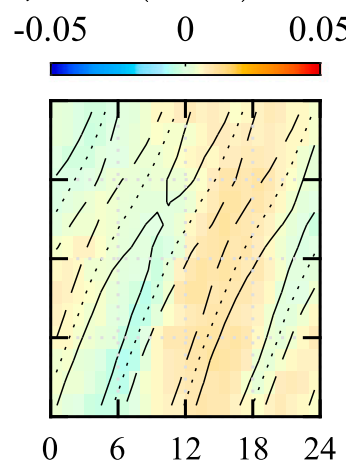

(h) (d) (samples)

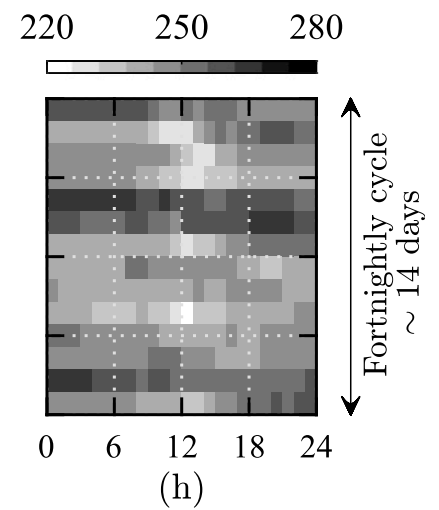

(h) (samples)

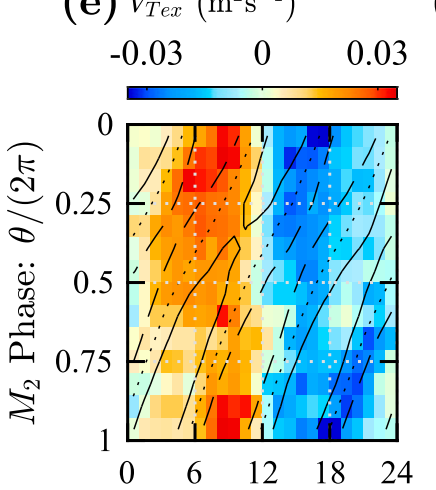

(f) $V_{B e x}\left(\mathrm{~m}^{2} \mathrm{~s}^{-1}\right)$

(g) $\Gamma_{e x}\left(\mathrm{~m}^{2} \mathrm{~s}^{-1}\right)$
0

0.04

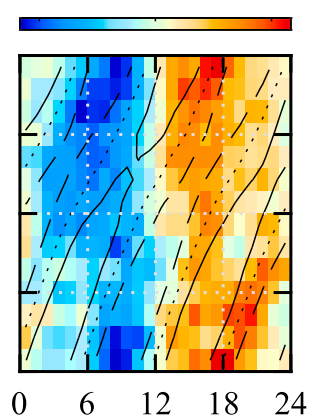

Diurnal phase (h)

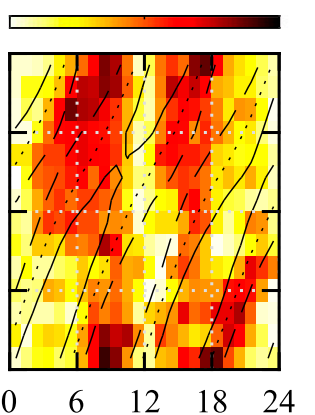

Diurnal phase (h)

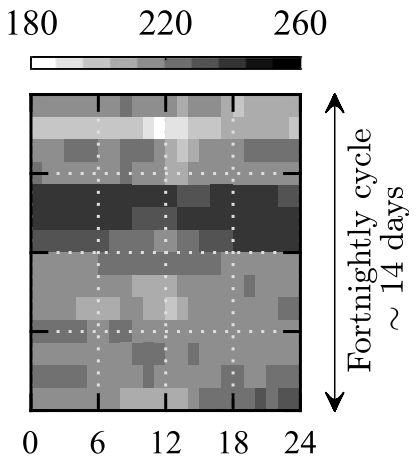

Diurnal phase (h)

FIG. 4. LSP $^{2}$ for KNO observations. (top) Ensemble averages for (a) tidal amplitude $\eta$, (b) depth-averaged alongshore velocity $\langle u\rangle_{D}$, and (c) depth-averaged cross-shore velocity $\langle v\rangle_{D}$, in terms of $\mathrm{M}_{2} /$ diurnal phase. Contour lines for tidal amplitude, $\eta=-0.1,0,0.1$, are overlaid in each panel. (d) Number of 20-min-averaged values contributing to each ensemble. (bottom),(e) Top-layer cross-shore transport $V_{\text {Tex }}$, (f) bottom-layer cross-shore transport $V_{\mathrm{Bex}}$, and (g) total cross-shore transport $\Gamma_{\text {ex }}$ with contour lines for $\eta$ overlaid in each panel. (h) Number of 20-min-averaged values contributing to each ensemble. Kona wind events are excluded from the ensemble averages. Note spring tide (full/new moon) occurs at $\theta / 2 \pi \approx 0.8$ with neap tide (first/third quarter) at $\theta / 2 \pi \approx 0.3$.

flow as $\langle u\rangle_{D}$ crosses from negative to positive, so that the flow rotates counterclockwise over the tidal cycle.

Figures $4 \mathrm{e}-\mathrm{g}$ show ensemble-averaged cross-shore exchange quantities in the $\mathrm{LSP}^{2}$ : Fig. $4 \mathrm{e}$ top-layer crossshore transport, $V_{\mathrm{Tex}}=\left\langle v_{\mathrm{ex}}\right\rangle_{\mathcal{L}_{T}} \mathcal{L}_{T} ;$ Fig. $4 \mathrm{f}$ bottom-layer cross-shore transport, $V_{\mathrm{Bex}}=\left\langle v_{\mathrm{ex}}\right\rangle_{\mathcal{L}_{B}} \mathcal{L}_{B}$; and Fig. $4 \mathrm{~g}$ total cross-shore exchange $\Gamma_{\text {ex }}$. The total exchange, defined here as the sum of the layer transports magnitudes, $\Gamma_{\mathrm{ex}}=\left|V_{\mathrm{Tex}}\right|+\left|V_{\mathrm{Bex}}\right|$, provides a measure of the baroclinic contribution to the cross-shore flow. Tidal amplitude contours are overlaid in the three fields. Since our focus here is on diurnal processes, strong, onshore Kona wind events (evident in the red regions in Fig. 2c) are excluded in the ensemble averaging, although results obtained using the full dataset (not shown) are not notably different. Both the top-layer and bottom-layer cross-shore transport show the predominantly diurnal pattern associated with the thermally driven cross-shore flow. The observed diurnal phase shift is consistent with the unsteady buoyancy, baroclinic/diffusive dynamic regime where $V_{\text {Tex }}>0$ during the cooling phase response $(\sim 0-12 \mathrm{~h})$, and $V_{\text {Tex }}<0$ during the heating phase response $(\sim 12-24 \mathrm{~h})$ with an opposite pattern for $V_{\text {Bex }}$.

The layer transports are subject to notable changes as a function of $\mathrm{M}_{2}$ phase, especially during the early cooling response $(0-6 \mathrm{~h})$ and late heating response (18-24h). The time for flow reversal shows significant variability relative to the long-term diurnal average that changes signs at midnight (Fig. 2j). The phasing and amplitude for the peak in baroclinic transport for each layer also vary with lunar phase for both cooling and heating responses. These features are further highlighted in the total cross-shore exchange $\Gamma_{\text {ex }}$ (Fig. 4c).

Comparing Figs. $4 \mathrm{~b}$ and $4 \mathrm{e}-\mathrm{g}$ more carefully, regions of weaker alongshore velocities appear to be correlated with stronger cross-shore transport, and vice versa. 
These data suggest that $\mathrm{M}_{2}$-driven alongshore currents actively lead to fortnightly variability in the diurnal cross-shore exchange.

\section{Theoretical model}

\section{a. Scaling}

We focus on describing the primary forcing mechanisms that drive and perturb cross-shore flows at diurnal time scales. The natural time scale for the cross-shore flow is $t \sim \mathcal{T}_{S}$, which reflects the period for variations in surface buoyancy fluxes. We will consider a scaling analysis at a particular cross-shore location with a depth $D_{0}$. Here, it is assumed that the sea surface height variation $\eta$ is negligible compared to the depth $D_{0}$ and that the bed slope is small $(\beta \ll 1)$. Additionally, we assume that length scales associated with alongshore variations in velocity, buoyancy, and bathymetric changes are large relative to the excursion of periodic alongshore currents, so that $\partial_{x}(\mathbf{v}, b) \approx 0$. Buoyancy is defined as $b=-\alpha g\left(T-T_{0}\right)$, where $T, T_{0}$, and $\alpha$ are the water temperature, reference water temperature, and the thermal expansion coefficient, respectively, and $g$ is the gravitational acceleration. Variations in buoyancy are thus controlled purely by changes in temperature. Effects associated with interaction of Stokes drift with planetary and local vorticity (Hasselmann 1970; Lentz et al. 2008) are neglected here since these are not expected to play a role at $\mathrm{KNO}$, though these may be important in general. We will revisit this in section 5.

The cross-shore and vertical velocity scales, given by $V_{0}$ and $W_{0}$, respectively, are determined by the dominant cross-shore dynamical balances (Monismith et al. 2006). From the continuity equation, $\partial_{y} v+\partial_{z} w=0$, it follows that $W_{0} \sim \beta V_{0}$. The alongshore velocity scale is given by $U_{0}$. We will assume that the alongshore flow works as a forcing mechanism in the cross-shore momentum balance.

Following observations and analysis for Kilo Nalu (Molina et al. 2014), we consider a baseline cross-shore flow with unsteady buoyancy, hydrostatic vertical momentum, and baroclinic/diffusive cross-shore momentum balances:

$$
\frac{\partial b}{\partial t} \sim \frac{\partial \mathcal{F}}{\partial z}, \quad \frac{1}{\rho_{0}} \frac{\partial p}{\partial z} \sim b, \quad \frac{1}{\rho_{0}} \frac{\partial p}{\partial y} \sim \bar{v}_{e} \frac{\partial^{2} v}{\partial z^{2}},
$$

where $\bar{v}_{e}$ is a spatially uniform reference eddy viscosity. The buoyancy flux is modeled as

$$
\frac{\partial \mathcal{F}}{\partial z}=-\frac{\alpha g H(t)}{\rho_{0} c_{p} D(y)}
$$

(e.g., Fischer et al. 1979), where $H(t)$ is the unsteady net surface heat flux, $\rho_{0}$ is the reference density of the water, $c_{p}$ is the heat capacity, and $D(y)$ is the depth at a distance $y$ from the shoreline. This parameterization assumes that the net surface heat flux is uniformly distributed over the water column, which is a reasonable approximation for shallow forereef systems with high vertical turbulent diffusivity. In this limit, we define the buoyancy, the pressure, and the cross-shore velocity scales in terms of integral quantities determined by a reference surface heat flux $H_{0}$ :

$$
\begin{aligned}
b_{0} & =\frac{\alpha g H_{0} \mathcal{T}_{s}}{\rho_{0} c_{p} D_{0}}, \\
p_{0} & =\frac{\alpha g H_{0} \mathcal{T}_{s}}{c_{p}}, \quad \text { and } \\
V_{0} & =\frac{\beta \alpha g H_{0} \mathcal{T}_{s} D_{0}}{\rho_{0} c_{p} \bar{\nu}_{e}}
\end{aligned}
$$

Following the scaling above, we can identify the following parameters to describe the flow regimes:

$$
\begin{aligned}
\mathrm{Gr}_{\beta} & =\frac{\beta^{2} \alpha g H_{0} \mathcal{T}_{s} D_{0}^{2}}{\rho_{0} c_{p} \bar{\nu}_{e}^{2}}, \\
C_{b} & =\frac{\rho_{0} c_{p} f U D_{0}}{\beta \alpha g H_{0} \mathcal{T}_{s}}, \quad \text { and } \\
S_{t} & =\frac{D_{0}^{2}}{\bar{\nu}_{e} \mathcal{T}_{s}}
\end{aligned}
$$

where $\mathrm{Gr}_{\beta}$ is a Grashof number representing the ratio of the buoyancy to turbulent diffusion. We define the Coriolis buoyancy parameter $C_{b}$ as the ratio of the Coriolis force to buoyancy. The Strouhal number $S_{t}$ is the ratio of the unsteady oscillatory force to the turbulent diffusion. The wind stress $\tau_{w}$ enters the problem through the surface boundary condition for the horizontal momentum balance and introduces an additional dimensionless parameter that represents the ratio of wind forcing to buoyancy effects:

$$
\mathcal{W}_{b}=\frac{c_{p} \tau_{w}}{\beta g \alpha H_{0} \mathcal{T}_{s}} .
$$

The baseline eddy viscosity can be expressed as $\bar{v}_{e} \sim q D_{0}$, where $q$ is a suitable turbulent velocity scale. While multiple sources of turbulence can contribute to vertical momentum diffusion in coastal environments, we will assume for the moment that the primary source is controlled by the alongshore flow. We therefore 
define $q$ using the bottom shear stress velocity $u_{*}$ associated with the alongshore flow.

To study the effect of the alongshore flow on the crossshore exchange through its influence on turbulent transport, we make use of a spatially constant eddy viscosity, modeled as a steady component plus a timedependent perturbation:

$$
v_{e}=\bar{v}_{e}[1+\gamma \mathcal{G}(t)]
$$

where $\gamma$ represents the relative magnitude of the perturbation and $\mathcal{G}$ is a function that captures the temporal structure of the turbulent transport.

Using nondimensional variables $\tilde{y}=y /\left(D_{0} / \beta\right), \tilde{z}=z / D_{0}$, $\tilde{t}=t / \mathcal{T}_{s}, \quad \tilde{\boldsymbol{v}}=\boldsymbol{v} / V_{0}, \quad \tilde{u}=u / U_{0}, \quad \tilde{w}=w /\left(\beta V_{0}\right), \quad \tilde{b}=b / b_{0}$, and $\tilde{p}=p / p_{0}$, along with the scaling quantities in Eq. (5), and considering an eddy viscosity given by Eq. (8), the equations of motion are given by the following:

$$
\begin{aligned}
& S_{t} \frac{\partial \tilde{v}}{\partial \tilde{t}}+\operatorname{Gr}_{\beta}\left(\tilde{v} \frac{\partial \tilde{v}}{\partial \tilde{y}}+\tilde{w} \frac{\partial \tilde{v}}{\partial \tilde{z}}\right)+C_{b} \tilde{u}=\left[-\frac{\partial \tilde{p}}{\partial \tilde{y}}+\frac{\partial^{2} \tilde{v}}{\partial \tilde{z}^{2}}\right]+\gamma \mathcal{G} \frac{\partial^{2} \tilde{v}}{\partial \tilde{z}^{2}}+\beta^{2}(1+\gamma \mathcal{G}) \frac{\partial^{2} \tilde{v}}{\partial \tilde{y}^{2}} \\
& S_{t} \beta^{2} \frac{\partial \tilde{w}}{\partial \tilde{t}}+\beta^{2} \operatorname{Gr}_{\beta}\left(\tilde{v} \frac{\partial \tilde{w}}{\partial \tilde{y}}+\tilde{w} \frac{\partial \tilde{w}}{\partial \tilde{z}}\right)=\left[-\frac{\partial \tilde{p}}{\partial \tilde{z}}+\tilde{b}\right]+\beta^{2}(1+\gamma \mathcal{G})\left(\beta^{2} \frac{\partial^{2} \tilde{w}}{\partial \tilde{y}^{2}}+\frac{\partial^{2} \tilde{w}}{\partial \tilde{z}^{2}}\right), \\
& {\left[\frac{\partial \tilde{b}}{\partial \tilde{t}}\right]+\frac{\mathrm{Gr}_{\beta}}{S_{t}}\left(\tilde{v} \frac{\partial \tilde{b}}{\partial \tilde{y}}+\tilde{w} \frac{\partial \tilde{b}}{\partial \tilde{z}}\right)=\left[\frac{\partial \tilde{\mathcal{F}}}{\partial \tilde{z}}\right]+\frac{\beta^{2}}{\operatorname{Pr}_{t} S_{t}} \frac{\partial^{2} \tilde{b}}{\partial \tilde{y}^{2}}, \quad \text { and }} \\
& {\left[\frac{\partial \tilde{v}}{\partial \tilde{y}}+\frac{\partial \tilde{w}}{\partial \tilde{z}}\right]=0}
\end{aligned}
$$

The effects of shortwave radiation and turbulent mixing are both included in the net vertical buoyancy flux $\tilde{\mathcal{F}}$ in the buoyancy equation [Eq. (9c)]. The term $\left(\operatorname{Pr}_{t} S_{t}\right)^{-1}$ represents a dimensionless diffusion coefficient, where $\operatorname{Pr}_{t}$ is the turbulent Prandtl number.

We will examine values of the dimensionless parameters in Eqs. (6) and (8) that are typical for KNO in section 4. For now we will proceed with the assumption that the zeroth-order flow follows the dynamic balances identified at KNO by Molina et al. (2014), as given in Eq. (3) and by the terms in square brackets in Eq. (9). To explore the effects of tidally driven alongshore currents on cross-shore variability, we therefore will focus the analysis on a hydrostatic linear regime and will examine the role of the first-order linear perturbations in Eqs. (9), neglecting terms at order $\beta^{2}$ and higher. Perturbations to the baseline thermally driven crossshore circulation will include 1) the tidally driven Coriolis acceleration, 2) temporal variations in the effective diffusivity, and 3) the cross-shore wind stress acting on the surface.

\section{b. Linear, hydrostatic regime}

We seek solutions for the simplified linear, hydrostatic equations of motion:

$$
\begin{aligned}
S_{t} \frac{\partial \tilde{v}}{\partial \tilde{t}}+C_{b} \tilde{u} & =-\frac{\partial \tilde{p}}{\partial \tilde{y}}+\frac{\partial^{2} \tilde{v}}{\partial \tilde{z}^{2}}+\gamma \mathcal{G} \frac{\partial^{2} \tilde{v}}{\partial \tilde{z}^{2}}, \\
0 & =-\frac{\partial \tilde{p}}{\partial \tilde{z}}+\tilde{b} \\
\frac{\partial \tilde{b}}{\partial \tilde{t}} & =\frac{\partial \tilde{\mathcal{F}}}{\partial \tilde{z}}, \quad \text { and } \\
\frac{\partial \tilde{v}}{\partial \tilde{y}}+\frac{\partial \tilde{w}}{\partial \tilde{z}} & =0 .
\end{aligned}
$$

The alongshore velocity $\tilde{u}(z, t)$ in Eq. (10a) is specified from observations or from a solution to the alongshore momentum balance that is considered uncoupled to the cross-shore flow. The time-dependent component for the eddy viscosity in Eq. (8) is then modeled based on the magnitude of the depth-averaged alongshore flow:

$$
\mathcal{G}=\left|\langle\tilde{u}\rangle_{D}\right|-\mathcal{G}_{0},
$$

where $\mathcal{G}_{0}$ is the time average of $\left|\langle\tilde{u}\rangle_{D}\right|$. For an alongshore flow varying at an $\mathrm{M}_{2}$ frequency, the timevariable fluctuation thus influences the baseline flow at a frequency of $2 \mathrm{M}_{2}$ and the mean eddy viscosity is unchanged. 
The heat flux and wind are modeled by simple sinusoidal functions that capture the time-averaged diurnal patterns observed at KNO:

$$
\begin{aligned}
\frac{\partial \tilde{\mathcal{F}}}{\partial \tilde{z}} & =-\frac{H(\tilde{t})}{H_{0} \tilde{y}}=\frac{\cos (2 \pi \tilde{t})}{\tilde{y}}, \quad \text { and } \\
\tilde{F}_{w}(\tilde{t}) & =A+B \sin \left(2 \pi \tilde{t}+\phi_{w}\right) .
\end{aligned}
$$

The phasing is such that $\tilde{t}=0$ at midnight. In Eq. (12a), we assume that the heat flux is distributed uniformly over the depth. We will discuss the implications and limitations associated with this assumption later. For the cross-shore wind velocity in Eq. (12b), the constants $A$ and $B$ and the wind phase $\phi_{w}$ are estimated using the time-averaged diurnal profile in Fig. 2d.

Equations (10) are subject to boundary conditions for the velocity given by

$$
\begin{aligned}
\left.\tilde{\boldsymbol{v}}\right|_{\tilde{z}=-\tilde{y}} & =0, \\
\left.(1+\gamma \mathcal{G}) \frac{\partial \tilde{\boldsymbol{v}}}{\partial \tilde{z}}\right|_{\tilde{z}=0} & =\mathcal{W}_{b} \tilde{F}_{w}, \quad \text { and } \\
\int_{\tilde{y}}^{0} \tilde{v} d \tilde{z} & =0,
\end{aligned}
$$

corresponding to no slip on the bottom, wind shear stress on the surface, and null net transport, respectively.

Considering harmonic solutions to Eq. (10c) using Eq. (12a), we obtain the baseline buoyancy field:

$$
\tilde{b}=\frac{\sin (2 \pi \tilde{t})}{2 \pi \tilde{y}}
$$

This buoyancy distribution results in the cross-shore baroclinic pressure gradient that drives the baseline cross-shore circulation.

The velocity field is obtained using a perturbation series solution, assuming

$$
S_{t} \sim W_{b} \sim C_{b} \sim \gamma \ll 1
$$

so that the velocity can be expressed as

$$
\tilde{\boldsymbol{v}}=\tilde{\boldsymbol{v}}^{(0)}+S_{t} \tilde{\boldsymbol{v}}^{(1)}+\ldots
$$

Details of the full solution are outlined in appendix A. At zeroth order, the baseline solution is obtained from a balance between a cross-shore baroclinic gradient and vertical diffusion, characterized by a cubic velocity profile:

$$
\tilde{\boldsymbol{v}}^{(0)}=-\frac{\tilde{y}}{96 \pi}\left\{8\left(\frac{\tilde{z}}{\tilde{y}}\right)^{3}-9\left(\frac{\tilde{z}}{\tilde{y}}\right)^{2}+1\right\} \sin (2 \pi \tilde{t}) .
$$

This is the viscous response obtained by Farrow and Patterson (1993) and represents a time-modulated version of the steady-state solution found by Cormack et al. (1975) for the core flow region of a differentially heated shallow rectangular basin. The solution neglects inertia and so its validity is limited to the nearshore region where water depth is smaller than a diffusive length $\sqrt{\nu_{e} \mathcal{T}_{s}}$, as evident in the growth of the velocity with cross-shore distance $\tilde{y}$.

The effects of the linear perturbations are represented in the solution at $O\left(S_{t}\right)$, detailed in appendix A. The first-order solution is then given by

$$
\begin{aligned}
\tilde{\boldsymbol{v}}^{(1)}= & \int_{\tilde{z}}^{0} \int_{\tilde{z}}^{0}\left(\frac{\partial \tilde{\boldsymbol{v}}^{(0)}}{\partial \tilde{t}}\right) d \tilde{z} d \tilde{z}+\frac{C_{b}}{S_{t}} \int_{\tilde{z}}^{0} \int_{\tilde{z}}^{0} \tilde{u} d \tilde{z} d \tilde{z} \\
& -\frac{\gamma}{S_{t}} \mathcal{G} \tilde{\boldsymbol{v}}^{(0)}+C_{1} \frac{\tilde{z}^{2}}{2}+C_{2} \tilde{z}+C_{3},
\end{aligned}
$$

where the functions $C_{1}, C_{2}$, and $C_{3}$, given in appendix $\mathrm{A}$, are obtained from the boundary conditions.

The full solution can be written in the following form:

$$
\tilde{v}=\tilde{v}_{v}+S_{t} \tilde{v}_{u}+\mathcal{W}_{b} \tilde{v}_{w}+C_{b} \tilde{v}_{c}+\gamma \tilde{v}_{t}+\ldots
$$

Here, the baseline velocity field is given by the zerothorder solution, $\tilde{v}_{v}=v^{(0)}$. The first-order solution from Eq. (18) is represented in terms of the four perturbation components. Expressions for each linear velocity contribution are outlined in appendix A.

For consistency with the constant eddy viscosity assumption, the alongshore flow is modeled as a Stokes boundary layer with a free surface, driven by a barotropic tidal pressure gradient (see solution in appendix B):

$$
\tilde{u}(\tilde{z}, \tilde{t})=\Re\left(\left\{\frac{1}{1-\tilde{\delta}_{\nu} \frac{(i-1)}{2} \tanh \left(\frac{i+1}{\tilde{\delta}_{\nu}}\right)}\right\}\left(1-\frac{\cosh \left\{\frac{(i+1)}{\tilde{\delta}_{\nu}} \tilde{z}\right\}}{\cosh \left\{\frac{(i+1)}{\tilde{\delta}_{\nu}}\right\}}\right) \exp \left(2 \pi i \frac{\omega_{u}}{\omega_{s}} \tilde{t}-i \phi_{u}\right)\right) .
$$


(a)

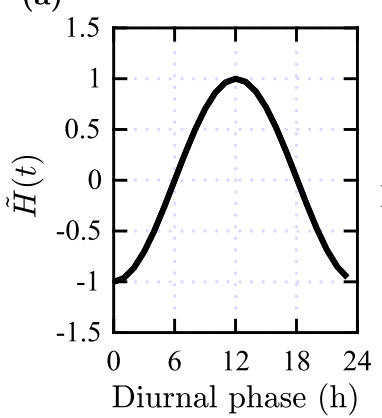

(b)

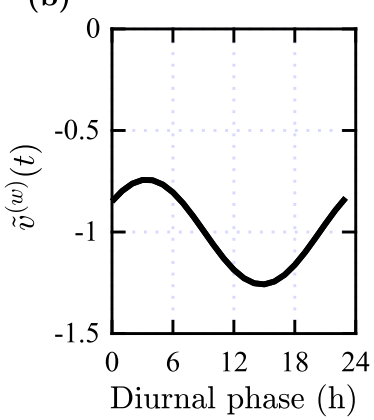

(c)

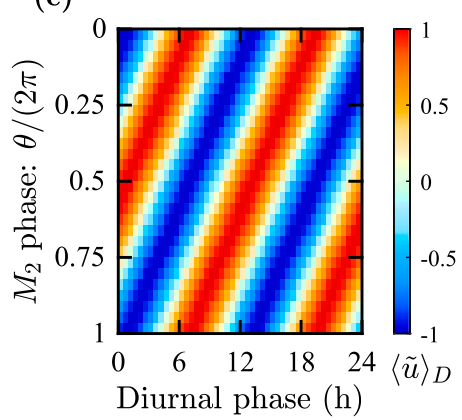

(d)

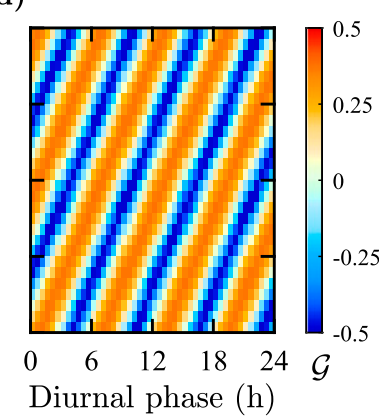

FIG. 5. (a) Modeled surface heat flux diurnal profile; (b) modeled cross-shore wind component diurnal profile; (c) depth-averaged theoretical alongshore flow $\langle\tilde{u}\rangle_{D}$, in terms of $\mathrm{M}_{2}$ and the diurnal phase; and (d) theoretical time-dependent eddy viscosity $\mathcal{G}$.

Here $\omega_{s}$ is the diurnal frequency, $\omega_{u}$ and $\phi_{u}$ are the frequency and phase of the alongshore flow, and $\tilde{\delta}_{\nu}=\sqrt{2 \nu_{e} /\left(\omega_{u}\right)} / D_{0}$ is the dimensionless Stokes boundary layer thickness. We use $\omega_{u}=\omega_{\mathrm{M}_{2}}$ with the phase $\phi_{u}=\phi_{\mathrm{M}_{2}}$ chosen to match the long-term observations at KNO. The solution yields a depth-averaged alongshore velocity that varies between \pm 1 . We choose $\tilde{\delta}_{\nu}=1$ for now, consistent with the dominant role of vertical turbulent diffusion in the momentum balance. The alongshore velocity in Eq. (20) then specifies the timedependent eddy viscosity in Eq. (11) with $\mathcal{G}_{0}=2 / \pi$. The Stokes boundary layer solution in Eq. (20) will be a poor approximation for a realistic, turbulent boundary layer where the effective eddy viscosity varies with depth. We will revisit this when we examine the application of the theoretical model for $\mathrm{KNO}$ in section $4 \mathrm{a}$.

Figure 5 shows the temporal structure of the forcing components in Eqs. (11)-(12) in terms of their respective time scales.

From each theoretical cross-shore velocity contribution in Eq. (19), we compute the upper layer transport as follows:

$$
\tilde{V}_{(\cdot), \operatorname{Tex}}=\int_{-\tilde{\mathcal{L}}}^{0} \tilde{v}_{(\cdot)} d \tilde{z}
$$

where $\tilde{\mathcal{L}}$ is the time-varying nondimensional interface depth given by the zero crossing in the cross-shore velocity profile. Figure 6 shows the upper-layer cross-shore transport components mapped onto the $\mathrm{LSP}^{2}$ as for the observational data in Fig. 4. Transport components for the bottom layer have the same temporal structure but with opposite signs. Because their corresponding forcing functions are purely diurnal, $\tilde{V}_{\nu}, \tilde{V}_{u}$, and $\tilde{V}_{w}$ do not vary with varying $\mathrm{M}_{2}$ phase. In contrast, solutions for $\tilde{V}_{c}$ and $\tilde{V}_{t}$ vary with lunar phase, reflecting the $\mathrm{M}_{2}$ contribution in the alongshore flow.

The baseline thermally driven cross-shore transport $\tilde{V}_{\nu \text {,Tex }}$ (Fig. 6a) has a daily circulation pattern that is in quadrature with the surface buoyancy flux cycle with temporal flow reversals occurring at $t=0 \mathrm{~h}$ and $t=12 \mathrm{~h}$. The unsteady cross-shore transport $\tilde{V}_{u \text {,Tex }}$ (Fig. 6b) results from a first-order balance between the unsteady component of the baseline solution and a perturbation vertical momentum flux and therefore follows a purely diurnal phase dependence. Wind-driven cross-shore
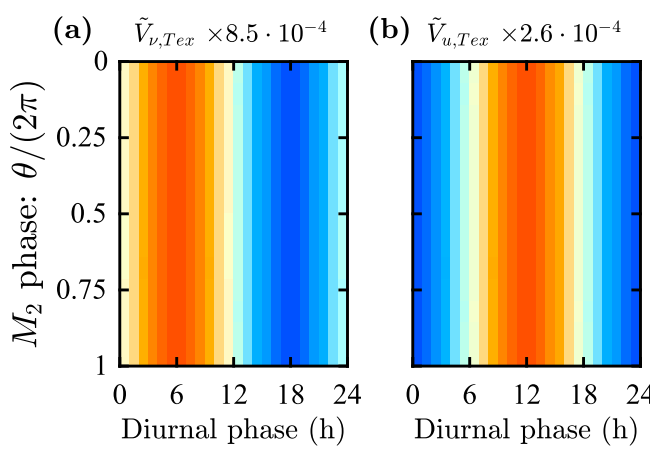

(c) $\tilde{V}_{w, T e x} \times 10^{-2}$

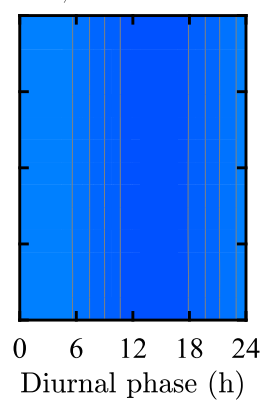

(d) $\tilde{V}_{c, T e x} \times 1.9 \cdot 10^{-3}$

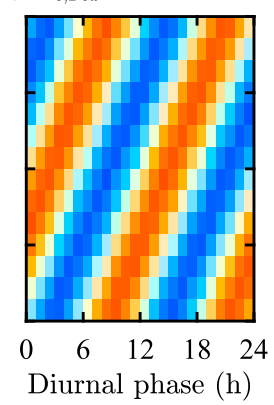

(e) $\tilde{V}_{t, T e x} \times 3.9 \cdot 10^{-4}$

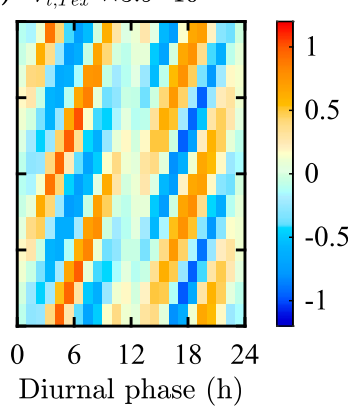

FIG. 6. Theoretical, linear top-layer transports $\left(\mathrm{LSP}^{2}\right)$ corresponding to the (a) baseline flow $\tilde{V}_{v}$, (b) unsteady flow component $\tilde{V}_{u}$, (c) wind-driven flow $\tilde{V}_{w}$, (d) Coriolis flow $\tilde{V}_{c\left(\mathrm{M}_{2}\right)}$, and (e) time-dependent eddy viscosity flow $\tilde{V}_{t}$. Theoretical bottom-layer transports have opposite signs. 
transport $\tilde{V}_{w, \text { Tex }}$ (Fig. 6c) similarly has a diurnal cycle in phase with the diurnal wind pattern, reinforcing the afternoon offshore surface layer flow. The Coriolis crossshore transport contribution $\tilde{V}_{c \text {,Tex }}$ (Fig. 6d) results from a balance between the Coriolis acceleration and a corresponding perturbation vertical momentum flux. This balance is forced by the tidally driven alongshore flow $\tilde{u}$ so that the diurnal phase of $\tilde{V}_{c \text {,Tex }}$ shifts following a fortnightly cycle. The cross-shore transport $\tilde{V}_{t, \text { Tex }}$ (Fig. 6e) is associated with a modulation of the turbulent momentum flux by a time-dependent component of the eddy viscosity [Eq. (A15d)]. Here, the modulation is related to the magnitude of the alongshore flow so the resulting perturbation occurs at twice the $\mathrm{M}_{2}$ frequency. The $\mathrm{LSP}^{2}$ shows that the Coriolis contribution and a time-dependent eddy viscosity perturbation can introduce temporal variability at diurnal time scales that depends on the phasing and strength of the alongshore currents.

\section{Results}

The long-term observations at KNO shown in Figs. 4e-g indicate that the cross-shore transport is primarily determined by the baseline diurnal component $\tilde{V}_{\nu}$, given by the solution shown in Fig. 6a. The linear coupling of the baseline, unsteady, and wind-driven flow components, $\tilde{V}_{\nu, \text { Tex }}+S_{t} \tilde{V}_{u, \text { Tex }}+\mathcal{W}_{b} \tilde{V}_{w, \text { Tex }}$, also leads to a purely diurnal cross-shore transport, but shifted with respect to the baseline pattern. The resulting phase shift will depend on the relative strength of the unsteady and winddriven contributions with respect to the baseline flow. Here, we focus on the effects of perturbations that drive diurnal variations as a function of the $\mathrm{M}_{2}$ phase.

To examine the role of the linear perturbation solutions in driving $\mathrm{M}_{2}$ variability in the cross-shore flow, we examine their superposition using estimates for the dimensionless parameters based on observations from KNO. The net surface heat flux $H(t)$ ranges from minimum values of $-200 \mathrm{~W} \mathrm{~m}^{-2}$ at night to maximum values of $600 \mathrm{~W} \mathrm{~m}^{-2}$ at midday at KNO. Wind stress values are estimated using a surface drag coefficient of $1.1 \times 10^{-2}$ with typical wind velocities at KNO of 2.5 to $4.5 \mathrm{~m} \mathrm{~s}^{-1}$. The bottom shear stress velocity $u_{*}$ is estimated based on depth-averaged alongshore velocities of $\langle u\rangle_{D} \approx$ $(0.5-1) \times 10^{-1} \mathrm{~m} \mathrm{~s}^{-1}$, with bottom drag coefficients $C_{D} \approx(1-2.5) \times 10^{-2}$ (Jones et al. 2008), yielding $u_{*} \approx$ $(0.1-1.6) \times 10^{-2} \mathrm{~m} \mathrm{~s}^{-1}$. The associated baseline eddy viscosity is calculated as $\nu_{t}=0.067 u * D_{0}$ based on the vertical average for a logarithmic turbulent boundary layer (Fischer et al. 1979). Table 1 lists typical values for the dimensionless parameters using these ranges for $H_{0}$ and $u *$, for $D_{0}=12 \mathrm{~m}$, and $\beta=3 \times 10^{-2}$.
TABLE 1. Range of magnitudes for parameters that influence the cross-shore flow at KNO.

\begin{tabular}{lc}
\hline \hline Parameter & Range \\
\hline $\mathrm{Gr}_{\beta}$ & $(0.07-50) \times 10$ \\
$\beta \mathrm{Gr}_{\beta}$ & $(0.02-16) \times 10$ \\
$C_{b}$ & $(0.09-3) \times 10^{-1}$ \\
$S_{t}$ & $(0.4-7) \times 10^{-1}$ \\
$\beta S_{t}$ & $(0.1-2) \times 10^{-2}$ \\
$\mathrm{Gr}_{\beta} S_{t}^{-1}$ & $(1.6-80) \times 10$ \\
$\mathcal{W}_{b}$ & $(0.1-1) \times 10^{-1}$ \\
\hline
\end{tabular}

The values in Table 1 generally reflect the zerothorder baseline hydrostatic, diffusive-momentum, unsteady buoyancy dynamic balances in Eq. (9), consistent with the long-term diurnal averaged flow observed by Molina et al. (2014). Relatively large values for $\mathrm{Gr}_{\beta}$, $\beta \mathrm{Gr}_{\beta}$, and $\mathrm{Gr}_{\beta} S_{t}^{-1}$ are notable, which appear to indicate a nonnegligible role for advection. As we will discuss further below, however, the contributions from the advective terms remain small even for finite values for the $\mathrm{Gr}_{\beta}$ parameters. In some limiting cases the advective terms may perturb the baseline balances, but under normal conditions the advective terms play at most a secondary role in the cross-shore dynamics.

As is evident from the solution in Fig. 6c, the wind perturbation provides a steady offset to the cross-shore transport with a diurnal component, weakening the cooling phase flow and strengthening the heating phase. The influence of the Coriolis contribution $\tilde{V}_{c, \text { Tex }}$ on the diurnal transport $\tilde{V}_{\text {Tex }}$ is illustrated in Fig. 7. The contribution of the Coriolis component can either reinforce or weaken the diurnal cross-shore transport depending of the $\mathrm{M}_{2}$ phase. This results in asymmetrical maxima for the cooling and heating phase that shift over the $\mathrm{M}_{2}$ cycle. The asymmetry in the pattern is intensified for increasing values of $C_{b}$.

Estimates for the diffusive perturbation parameter $\gamma$ are not available for KNO, but based on the dominance of the diffusive term in the baseline balance, we can anticipate that modulation in bottom-driven turbulence associated with the alongshore flow will have an important role in the cross-shore variability. To explore the effects of a time variation in diffusivity, we consider $\gamma=0$, $0.25,0.5$. Contributions due to unsteady acceleration, Coriolis, and wind are superposed on the baseline solution for the top-layer transport $\tilde{V}_{\text {Tex }}$ in Fig. 8, for $S_{t}=10^{-1}$, $\mathcal{W}_{b}=10^{-2}, C_{b}=10^{-1}$, along with the time-dependent turbulent component using the three values of $\gamma$.

The effects of time-variable bottom-driven turbulence are included in Figs. 8b,c for $\gamma=0.25$ and $\gamma=0.50$, corresponding to periodic variations in the effective eddy viscosity of $25 \%$ and $50 \%$ of the baseline value, respectively. The variations in eddy diffusivity lead to 

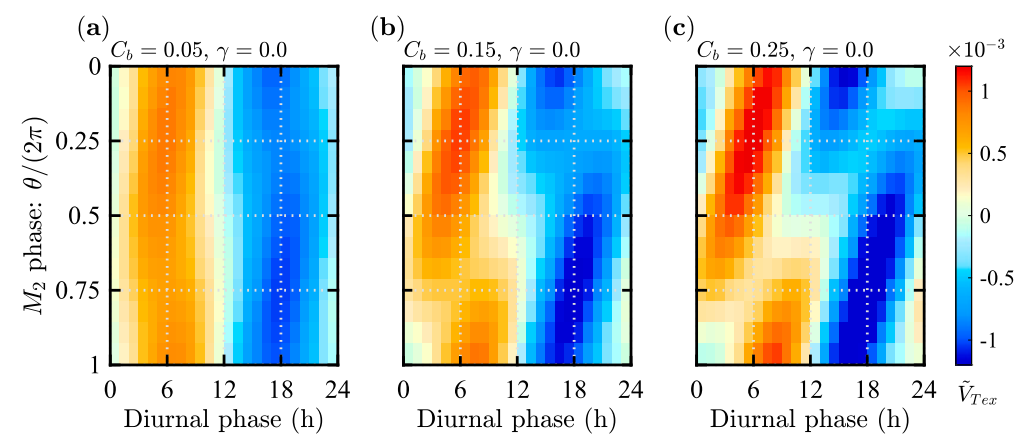

FIG. 7. Theoretical top-layer cross-shore transport $\tilde{V}_{\mathrm{Tex}}$ for $S_{t}=0.1, \mathrm{O}_{b}=0.01$, $\gamma=0$, and $C_{b}=$ (a) 0.05 , (b) 0.15 , and (c) 0.25 .

periodic weakening of the cross-shore transport every $T_{\mathrm{M}_{2}} / 2=6.21 \mathrm{~h}$. The linear combinations of the perturbation components thus can drive significant fortnightly variations in the $\mathrm{LSP}^{2}$ with patterns that begin to resemble those apparent in the observations (Fig. 4).

\section{a. Comparison with observations}

The ability of the simple linear analysis to capture some of the features apparent in the observed crossshore transport motivates a more detailed comparison with the KNO observations. For the purpose of comparison, the model is modified to use a more realistic alongshore velocity profile than the viscous flow solution given in Eq. (20). We assume, instead, that a fully developed, quasisteady turbulent boundary layer applies, given by a logarithmic velocity profile:

$\tilde{u}=\left\{\frac{\ln \left(\tilde{z}_{0}\right)}{\tilde{z}_{0}-1}-1\right\} \ln \left(\frac{1+\tilde{z}}{\tilde{z}_{0}}\right) \cos \left(2 \pi i \frac{\omega_{u}}{\omega_{s}} \tilde{t}-\phi_{u}\right)$,

where the parameter $z_{0}$ is a measure of the hydrodynamic roughness. Typical values for $z_{0}$ are of order $\sim 10^{-2} \mathrm{~m}$ for KNO and other reefs (Jones et al. 2008; Lentz et al. 2017), substantially smaller than the local depth $D_{0}$. Here, we set $z_{0}=10^{-2} \mathrm{~m}$ so $\tilde{z}_{0} \approx 10^{-3}$. The diurnal thermal exchange is subject to additional $\mathrm{LSP}^{2}$ perturbations that have thus far not been included in the theoretical model. At KNO, for example, the alongshore flow has a significant contribution at the $\mathrm{S}_{2}$ frequency (period $12 \mathrm{~h}, 33 \%$ of the variance; Fig. 2f) that will further modulate turbulent mixing. In addition, a number of other mechanisms can drive turbulent mixing including surface wind stress and convection. The former can be assumed to be fairly steady across the $\mathrm{LSP}^{2}$ given the relatively weak diurnal signal evident in Figs. $2 \mathrm{c}$ and $2 d$. This contribution factors into the baseline eddy viscosity. Convection, however, can be expected to play an important role in vertical mixing during the nightly cooling phase where surface fluxes result in unstable temperature gradients (Sevadjian et al. 2010).

We superpose an $\mathrm{S}_{2}$ tide constituent to the alongshore flow component so that $\tilde{u}=A_{\mathrm{M}_{2}} \tilde{u}_{\mathrm{M}_{2}}+A_{\mathrm{S}_{2}} \tilde{u}_{\mathrm{S}_{2}}$, with amplitudes $A_{\mathrm{M}_{2}} \approx 0.7$ and $A_{\mathrm{S}_{2}} \approx 0.3$ matching the relative magnitudes at KNO. Each component is assumed to contribute a velocity given by Eq. (22) with corresponding phases determined by fitting to the observed $\langle\tilde{u}\rangle_{D}$. This linear superposition leads to an improved approximation of the alongshore flow structure in the $\mathrm{LSP}^{2}$ in Fig. $4 \mathrm{~b}$.

We also incorporate a simple model for the nightly cooling-induced eddy viscosity in the global timedependent eddy viscosity function given in Eq. (8):
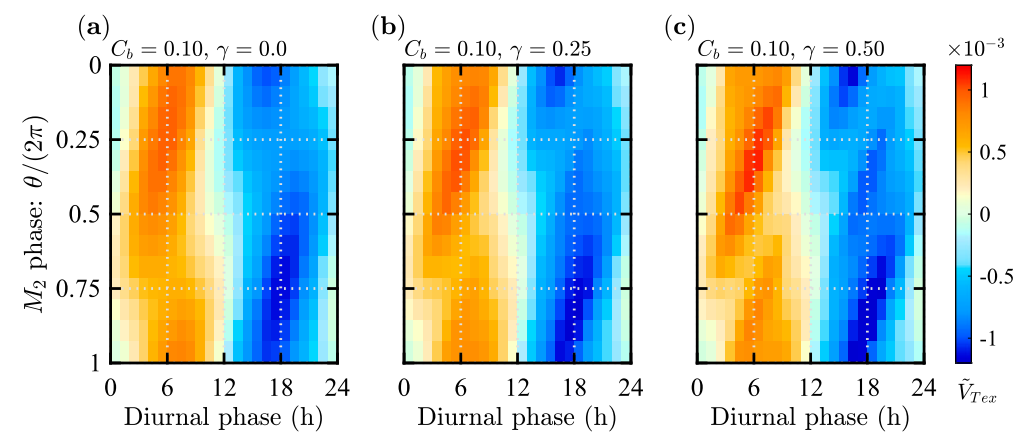

FIG. 8. Theoretical top-layer cross-shore transport $\tilde{V}_{\text {Tex }}$ for $S_{t}=0.1, \mathrm{O}_{b}=0.01, C_{b}=$ 0.1 , and $\gamma=$ (a) 0 , (b) 0.25 , and (c) 0.5 . 

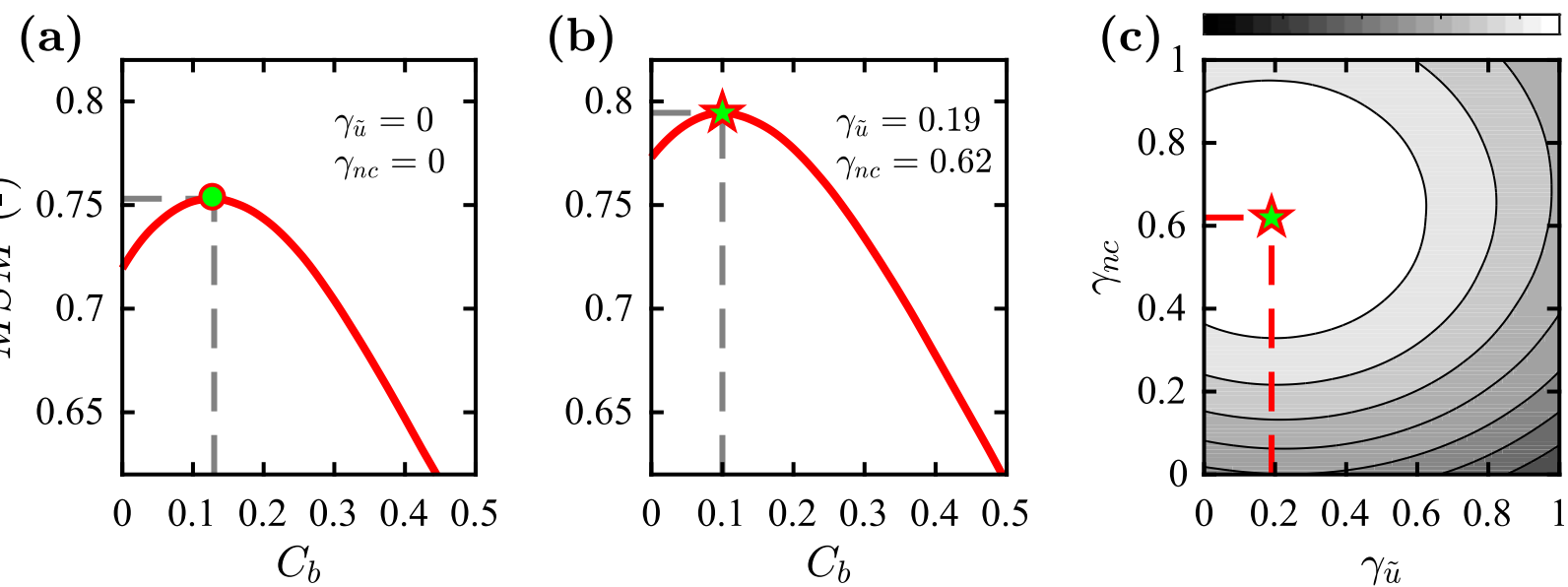

FIG. 9. MSM analysis for theoretical dimensionless total exchange $\tilde{\Gamma}_{\mathrm{ex}}$. (a) MSM as a function of $C_{b} \in[0,0.5]$, for $\gamma=0$. The green circle indicates maximum MSM $\left(\gamma_{\tilde{u}}=\gamma_{\mathrm{nc}}=0\right)$. (b) MSM as a function of $C_{b} \in[0,0.5]$, for the optimal values of the diffusivity parameters $\gamma$. (c) MSM for $\tilde{\Gamma}_{\text {ex }}$ as a function of $\gamma_{\tilde{u}}$ and $\gamma_{\mathrm{nc}}$, for the optimal value $C_{b} \approx 0.1$. The green star indicates maximum MSM for optimal $\gamma_{\tilde{u}}, \gamma_{\mathrm{nc}}$, and for optimal $C_{b}$, in (b) and (c), respectively. Optimal parameters: $\operatorname{MSM}\left(\tilde{\Gamma}_{\mathrm{ex}}\right) \approx 0.795$ for $\left(C_{b}, \gamma_{\tilde{u}}, \gamma_{\mathrm{nc}}\right) \approx(0.1,0.19,0.62)$.

$$
\gamma \mathcal{G}(\tilde{t})=\gamma_{\tilde{u}} \mathcal{G}_{\tilde{u}}(\tilde{t})+\gamma_{\mathrm{nc}} \mathcal{G}_{\mathrm{nc}}(\tilde{t})
$$

where the first term $\mathcal{G}_{\tilde{u}}$ is defined as in Eq. (11), but now such that $\tilde{u}$ results from the linear superposition of $\mathrm{M}_{2}$ and $\mathrm{S}_{2}$ tide constituents as described above. The second term $\mathcal{G}_{\text {nc }}$ represents the increment in eddy diffusivity driven by nightly natural convection. Here, we choose a simple analytical function to model this variation as

$$
\mathcal{G}_{\mathrm{nc}}(\tilde{t})=\Re\{\sqrt{\cos (2 \pi \tilde{t})}\} \text {. }
$$

To have a robust assessment of the theoretical results, we compare the modeled total exchange,

$$
\tilde{\Gamma}_{\mathrm{ex}}=\frac{\Gamma_{\mathrm{ex}}}{D_{0} V_{0}}
$$

with the observations, making use of the model skill metric (MSM) (Willmott 1981):

$$
\mathrm{MSM}=1-\frac{\sum\left|x_{\text {mod }}-x_{\mathrm{obs}}\right|^{2}}{\sum\left(\left|x_{\text {mod }}-\bar{x}_{\mathrm{obs}}\right|+\left|x_{\mathrm{obs}}-\bar{x}_{\mathrm{obs}}\right|\right)^{2}} .
$$

Here, $x_{\text {mod }}$ is the modeled field, to be compared with the observed field $x_{\text {obs }}$ and its mean, $\bar{x}_{\text {obs }}$. Values for MSM range between 0 and 1 , where MSM $\rightarrow 1$ implies better model performance.

Model values for $\tilde{\Gamma}_{\mathrm{ex}}$ are calculated using $S_{t}=10^{-1}$ and $\mathcal{W}_{b}=10^{-2}$ for the diurnal velocity components. We exclude the very near-surface and near-bed regions, where ADCP measurements are unavailable, from the theoretical analysis to facilitate the comparison between the observations and model. To focus on the temporal variability, both the modeled and observed transports are normalized by twice their $\mathrm{LSP}^{2}$ rms values.

Figure 9 summarizes results of the MSM analysis considering variations in Coriolis parameter and time-dependent eddy viscosity components. We first isolate the Coriolis contribution $\left(\gamma_{\tilde{u}}=\gamma_{\mathrm{nc}}=0\right)$, considering a range of values for $C_{b}$ between 0 and 1 , with the alongshore velocity given by Eq. (22), including both $\mathrm{M}_{2}$ and $\mathrm{S}_{2}$ tide constituents. Resulting variations in MSM, shown in Fig. 9a, are relatively weak since the bulk of the signal is diurnal, but show a clear maximum at $C_{b} \approx 0.1$, with $\mathrm{MSM} \approx 0.75$, improving from $\mathrm{MSM} \approx 0.72$ for $C_{b}=0$. Next, MSM is calculated over a range of values for $C_{b}, \gamma_{\tilde{u}}$, and $\gamma_{\mathrm{nc}}$, with an optimal value MSM $\approx 0.80$ obtained for $C_{b} \approx \gamma_{\tilde{u}}=0.19$ and $\gamma_{\mathrm{nc}}=0.62$. The optimal Coriolis parameter is largely unchanged by including $\gamma_{\tilde{u}}$ and $\gamma_{\mathrm{nc}}$ (Fig. 9b) and compares well with the estimated range for KNO in Table 1.

The increment of MSM associated with $\gamma_{\mathrm{nc}}$ suggests that the nightly convective turbulence is an important parameter for understanding the diurnal cross-shore exchange. We can estimate the convective vertical velocity magnitude driven by a cooling water surface following Fischer et al. (1979) as

$$
w_{\mathrm{nc}}=\left(\frac{\alpha g D_{0} H}{C_{p} \rho_{0}}\right)^{1 / 3} \text {. }
$$

Assuming that $w_{\mathrm{nc}}$ is a suitable turbulence velocity scale for night hours, the effective eddy viscosity can be estimated as $v_{t} \sim w_{\mathrm{nc}} D_{0}$. Using typical magnitudes for the 

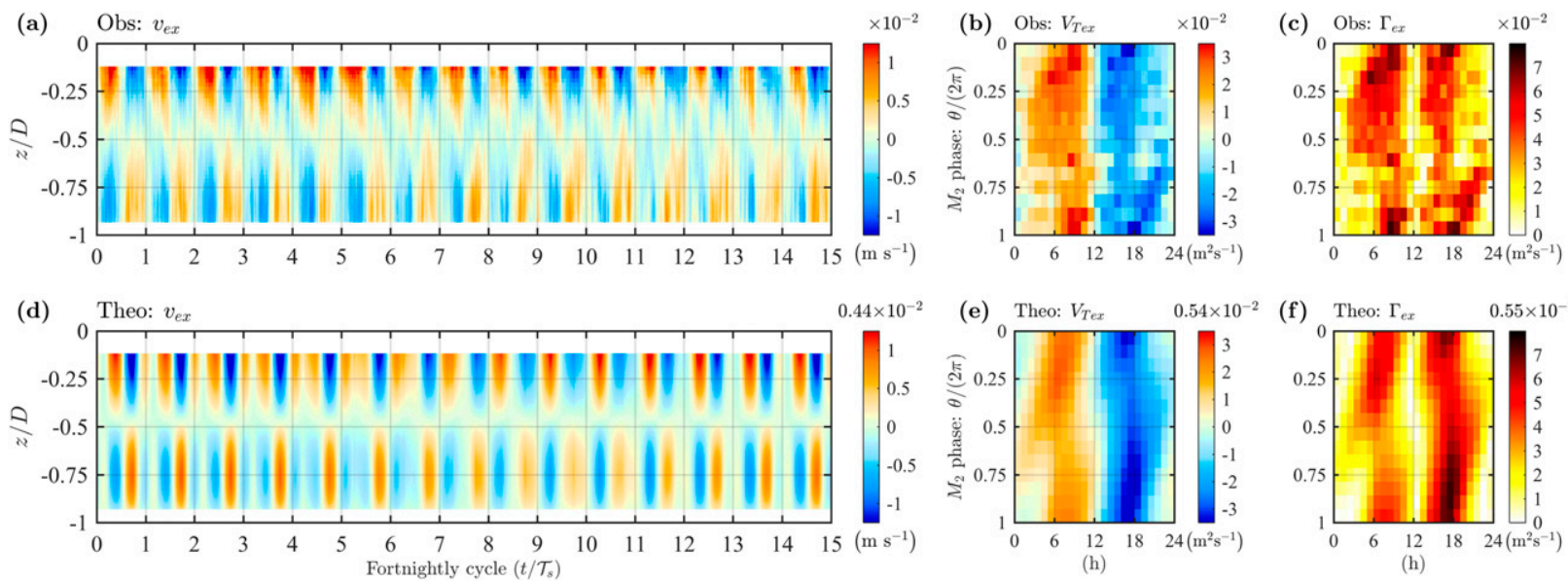

FIG. 10. Comparison of the theoretical model with observations. Observations: (a) $\mathrm{M}_{2}$-phase variation of $\tilde{v}$, along with (b) top-layer cross-shore transport $\tilde{V}_{\text {Tex }}$ and (c) total exchange $\tilde{\Gamma}_{\text {ex }}$. (d),(e),(f) As in (a)-(c), but for the theoretical results: incorporating $\mathrm{M}_{2}$ and $\mathrm{S}_{2}$ constituents and nightly convection in time-dependent eddy viscosity model.

net cooling phase heat flux, $H \approx-200 \mathrm{~W} \mathrm{~m}^{-2}$, then $w_{\mathrm{nc}} \approx$ $3 \times 10^{-2} \mathrm{~m} \mathrm{~s}^{-1}$, comparable with the shear stress velocity $u *$ adopted to estimate the magnitude of the effective eddy viscosity and the corresponding dimensionless parameters in Table 1 . We can thus expect comparable values for $\gamma_{\tilde{u}}$ and $\gamma_{\mathrm{nc}}$ during the cooling phase.

Figure 10 compares fortnightly cycle variability between observations at $\mathrm{KNO}$ and the theoretical results incorporating $\mathrm{M}_{2}$ and $\mathrm{S}_{2}$ constituents, along with the nightly convection effects in the time-dependent eddy viscosity model. Model parameters are based on the MSM analysis, optimizing the total exchange comparison $\left(C_{b}=0.10, \gamma_{\tilde{u}}=0.20\right.$, and $\left.\gamma_{\mathrm{nc}}=0.60\right)$. The theoretical results are given in dimensional form following the scaling outlined in section $3 \mathrm{a}$. Here we use $D_{0}=12 \mathrm{~m}$ as the vertical scale along with average magnitudes at KNO for the relevant parameters $\beta=3 \times 10^{-2}, H_{0}=$ $400 \mathrm{~W} \mathrm{~m}^{-2}, U_{0}=0.05 \mathrm{~m} \mathrm{~s}^{-1}$, and $\bar{\nu}_{e}=5 \times 10^{-3} \mathrm{~m}^{2} \mathrm{~s}^{-1}$, in the definition for $V_{0}$ [Eq. (5)].

The linear model effectively reproduces the main diurnal flow structure and its modulation as a function of $\mathrm{M}_{2}$ phase, including the amplification of the transport at the start and end of the fortnightly cycle. Despite averaging over numerous fortnightly cycles $(\sim 100)$, the observations in Fig. 10a include notable high-frequency fluctuations $(\sim 1 \mathrm{~h})$, especially in the bottom layer. These high-frequency features, which are not represented in the linear model, appear as well in the temporal variability in the $\operatorname{LSP}^{2}$ for $\tilde{\Gamma}_{\text {ex }}$ and $V_{\text {Tex }}$. The theory underestimates the magnitude of the crossshore transport by nearly a factor of 2 , likely associated with uncertainties in selecting appropriate values for the various parameters.

\section{Discussion and conclusions}

We have investigated fortnightly variability in diurnal thermally driven cross-shore circulation, focusing on the role of semidiurnal tidally driven alongshore flows. High variability in observations of cross-shore exchange at $\mathrm{KNO}$ in Oahu, Hawaii, provide a motivating example for examining these processes. The cross-shore transport at KNO shows a robust diurnal signal associated with an unsteady buoyancy/diffusive momentum balance (Molina et al. 2014). The cross-shore exchange shows significant variability relative to the diurnal signal as a function of the $\mathrm{M}_{2}$ phase, however, as highlighted in the lunar-solar phase-plane plots in Fig. 4.

The tidally driven alongshore flow plays a direct a role in the cross-shore exchange momentum balance via the Coriolis acceleration but also affects the cross-shore circulation indirectly via its influence on vertical turbulent diffusion. At KNO, the alongshore flow is dominated by an $\mathrm{M}_{2}$ contribution, so that the effects of these mechanisms will vary over the fortnightly cycle.

Using the long-term time-averaged diurnal dynamic balance at KNO as a baseline, we have formulated a linear theoretical model for baroclinic cross-shore flow driven at leading order by the surface heat flux, with contributions from the alongshore flow and cross-shore wind appearing as linear perturbations. Superposition of the idealized solutions for Coriolis and time-varying eddy viscosity perturbations are able to reproduce key aspects of the fortnightly variability as evident in Fig. 8. Modifying the model to consider a more realistic alongshore flow and considering effects of nightly convection lead to further improvements in comparisons 
with KNO observations. The ability of the theoretical approach to reproduce the general $\mathrm{LSP}^{2}$ patterns indicates that semidiurnal variations in the alongshore flow are effective in modulating the cross-shore flow via Coriolis and vertical turbulent transport mechanisms.

While fortnightly patterns for the exchange are effectively captured by the theoretical model, these account for a limited fraction of the overall variation in the cross-shore exchange. The diurnal pattern of the exchange at $\mathrm{KNO}$ and its variability is summarized in Fig. 11. The semidiurnal variability (green shading) computed over the ensemble-averaged $\mathrm{M}_{2}$ cycle, represents a nonnegligible portion $(\sim 20 \%)$ of the overall variation for the measurement period (January 2007-September 2011), although a large part of the variability remains unexplained.

For the KNO observations, we have defined the crossand alongshore directions based on the principal axes of the predominantly along-isobath tidal flow. A variation or error in the angle of the principal axes would result in alongshore variability appearing as a contribution to the cross-shore exchange, in phase with the alongshore flow, similar to the Coriolis component. Depending on the sign of the shift in the axis, this would appear in the same or opposing sense of the Coriolis contribution. From a consideration of the scaling and dimensionless parameters, the magnitude of the Coriolis contribution to the cross-shore velocity can be shown to be $f \Delta U_{z} \mathcal{T}_{s} S_{t}$, where $\Delta U_{z}$ is a measure of the top-to-bottom difference in alongshore velocity. The cross-shore contribution associated with a variation of the principal axes $\Delta \alpha$ is $\Delta U_{z} \sin \Delta \alpha$. These contributions thus have comparable magnitudes when $\Delta \alpha \sim \sin ^{-1}\left(f \mathcal{T}_{s} S_{t}\right)$. Using estimated values for KNO indicates that errors in the axes would need to be $\sim 20^{\circ}$ degrees to be of consequence, much larger than the $95 \%$ confidence limits on principal axes estimates $\left(\sim 0.8^{\circ}\right)$. Varying the coordinate system by $\pm 5^{\circ}$ resulted in changes in optimal MSM $C_{b}$ values of \pm 0.035 , for example. Variations in the alongshore flow direction can, nevertheless, introduce additional variability into cross-shore exchange.

The observations in Fig. 2 point toward other potentially important sources for variability including variations in surface heat flux, wind, and secondary tidal constituents. Larger spatial- and temporal-scale variations in water masses will also result in lateral transport of warm or cold water that can modify the thermal flow structure. The south shore of Oahu is also subject to strong internal tide forcing (Alford et al. 2006), which is intermittently manifested via cold-water intrusions (Pawlak et al. 2009; Smith et al. 2016). These events can interrupt and overwhelm the thermally driven cross-shore flow with important contributions of their own to exchange (Davis et al. 2008, Leichter et al. 2003). The influence of the

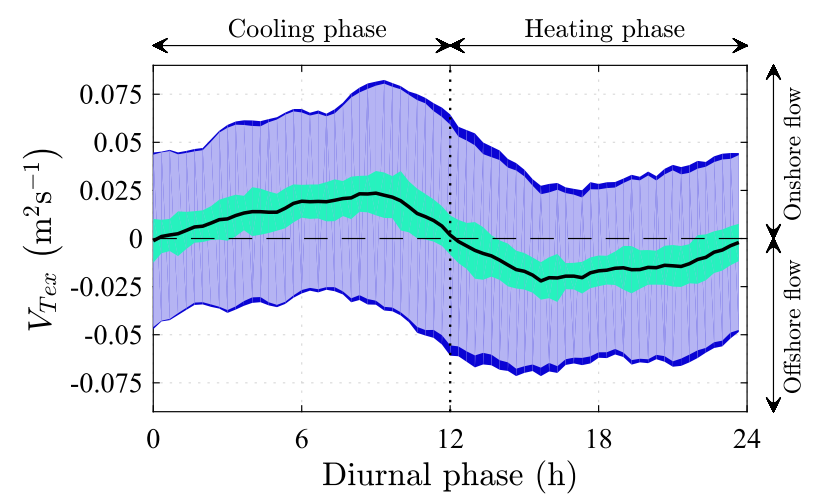

FIG. 11. Diurnal variation of top-layer cross-shore transport $V_{\text {Tex }}$ (solid black line), along with its standard deviation $\sigma_{V_{\mathrm{Tex}}}$ (darkest-blue shading, based on the full dataset; light-blue shading, excluding the Kona storm days). Green shading indicates the standard deviation of $V_{\text {Tex }}$ based on the ensemble-averaged $\mathrm{M}_{2}$ cycle $\sigma_{\mathrm{M}_{2}}$.

internal tide further modulates the alongshore flow, accounting for some of the variability apparent in Fig. 2e.

Surface waves can also affect cross-shore velocities well outside of the breaking region. In particular, a wave-induced stress resulting from Coriolis effects on wave motion (cf. Hasselmann 1970) can drive an Eulerian cross-shore flow (Lentz et al. 2008; Kumar and Feddersen 2017) that can contribute to variability in the observed cross-shore flow. The resulting flow largely balances the Stokes drift so that the net effect on the actual exchange is generally small. For KNO, Stokes drift effects are expected to be small owing to generally weak wave forcing and prevailing offshore winds that limit highfrequency wave contributions that would dominate these processes. A similar effect can arise from interaction of the Stokes drift with local vorticity. Since local vorticity may be associated with the alongshore flow, the resulting Eulerian flow could influence LSP ${ }^{2}$ variability for locations where Stokes drift can be expected to be important.

The theoretical solution used here considers a periodic heat flux that is uniformly distributed over the water column. This simplistic heating model is analytically convenient and provides an effective approximation for the cooling phase where vertical convection distributes heat rapidly. The uniform heat distribution is also a reasonable model for the heating phase in diffusively dominated systems like KNO, where turbulence keeps the water column well mixed or relatively weakly stratified (cf. Monismith et al. 2006; Molina et al. 2014; Herdman et al. 2015). Numerical studies that have adopted more realistic parameterizations, such as Beer's law, do not show notable changes in diffusive regimes (Farrow and Patterson 1994; Mao et al. 2009). However, this formulation is unlikely to be the most suitable for 
unsteady or nonlinear flow regimes or for deeper or more turbid regions.

The discrepancies between the model and the observations in the $\mathrm{LSP}^{2}$ are not unexpected given the assumptions used in the analysis. In particular, the assumption of a spatially uniform eddy viscosity is likely to limit the comparison. A uniform diffusivity would overestimate the role of turbulent diffusion near the surface and bottom and would underestimate its contribution in the interior. In addition, drag coefficients can vary in time as tidal flow varies relative to wave forcing (Grant and Madsen 1979) and, as shown recently by Lentz et al. (2017), with variations in water depth, thus modifying the effective vertical diffusion. Variations in turbulence with changing water depth are not included in the conceptual model, assuming $\eta \ll D$, although this may introduce variability at tidal time scales in very shallow regions where $\eta$ becomes important relative to the local average water depth.

The analysis further neglects any alongshore variability in velocity or temperature gradients. Observations by Molina et al. (2014), however, show that the cross-shore exchange structure at KNO varies with alongshore flow direction. We have also considered a purely cross-shore wind stress, while KNO winds have a persistent (westward) alongshore wind component that can affect the alongshore velocity profile and thus modulate the Coriolis contribution to the exchange. Coupling between the alongshore wind and currents might similarly modulate the fortnightly variability in the time-varying turbulent mixing.

There is considerable high-frequency variability that persists in the $\mathrm{LSP}^{2}$ (Figs. 4 and 10) despite averaging over a large number $(\sim 100)$ of fortnightly cycles. This may be associated with fortnightly internal tide activity, although analysis of events at KNO has not shown coherent internal tide forcing. It is more likely that the shorter time scales reflect the persistent directional variations in water properties identified by Molina et al. (2014).

The time-varying eddy viscosity associated with the alongshore flow represents a perturbation relative to a baseline average turbulent vertical diffusivity. The formulation in Eq. (8), along with Eq. (11), modulates this background value within the $\mathrm{LSP}^{2}$ via the alongshore flow. In the long-term average diurnal structure (i.e., Fig. 3), the baseline turbulent diffusivity represents an average of the time-variable eddy diffusivity along with other persistent contributions like convection and wind-driven turbulence. In both the diurnal and $\mathrm{LSP}^{2}$ ensemble averages, the effective background eddy diffusivity is also likely influenced by the general variability in the cross-shore flow, however. This variability smooths variations in the diurnal pattern and thus can appear as an enhanced background diffusive process in the $\mathrm{LSP}^{2}$ even at times when alongshore flow is weak.

In obtaining the linear solution, we have assumed that the nonlinear terms in Eq. (9) are negligible. This typically requires that $\mathrm{Gr}_{\beta} \ll 1$. Estimates for $\mathrm{Gr}_{\beta}$ (Table 1 ) yield values of order 10-100, which would appear to indicate significant advective contributions. Magnitudes for the dimensionless velocity [Eq. (17)] and the baseline terms are $\sim 10^{-3}$, however, so that the advective terms are $O\left(\mathrm{Gr}_{\beta} \times 10^{-6}\right)$ and thus remain negligible.

The fact that the scaling approach does not yield dimensionless velocities of $O(1)$ derives in part because the scaling analysis is based on the heat flux, and thus begins with the buoyancy equation. Several $O(>1)$ factors, neglected in deriving the velocity scaling in Eq. (5), are subsequently compounded in the solution resulting in the $1 / 96 \pi$ factor in Eq. (17). The dimensional velocity magnitudes obtained from the baseline solution are still somewhat small relative to the observed exchange velocities (Fig. 10). Farrow and Patterson (1993) found similar discrepancies and used larger values for $\tilde{v}_{e}$ to obtain comparable dimensional values. The higher $\tilde{v}_{e}$ value results in large $\mathrm{Gr}_{\beta}$, however. It is more likely that the low bias of the model velocities are associated with the use of a spatially uniform eddy viscosity, as noted above.

The perturbation approach adopted here to examine the effect of different mechanisms on the temporal variability of the baseline diffusive regime observed at KNO could similarly be applied for other linear baseline flow regimes considering the remaining mechanisms as perturbations. For instance, one could consider the scenario where Coriolis effects control the cross-shore exchange, and the thermal forcing appears as a perturbation.

Besides tropical coastal regions that are subject to high surface heat fluxes and tidal forcing, the theoretical framework presented here can be useful for analyzing buoyancy-driven cross-shore exchange in lentic aquatic systems, such as lakes and reservoirs. As an example, crossshore exchange in lakes can be perturbed by the diurnal winds (Farrow 2013), inertial oscillations (Farrow 2002), and also by periodic basin-scale internal waves and currents, such as Kelvin waves and gyre circulations.

Our results show the potential for tidally forced alongshore flows to drive systematic shifts in the crossshore exchange on tidal time scales. In regions where tidal flows are strong, the thermally driven cross-shore circulation can be significantly modified as a function of the local tidal cycle. One important limitation in assessing these effects is in the length of the observational time series. Numerous fortnightly cycles are needed to obtain a robust signal of the $\mathrm{M}_{2}$-driven diurnal variability across the $\mathrm{LSP}^{2}$, for example. The idealized theoretical framework can thus be useful in assessing these effects 
for general coastlines as well as for examining the role of additional tidal constituents and wind forcing.

Acknowledgments. The work here was carried out with support from the National Science Foundation via Awards OCE-1436254 and OCE-1436522. The authors are grateful to Sarah Giddings and Antonio Sanchez for meaningful discussions on the data interpretation and development of the theoretical model and to Oscar Sepúlveda Steiner for helpful comments on model skill assessments. The manuscript also benefited from feedback from two anonymous reviewers.

\section{APPENDIX A}

\section{Perturbation Series Solution}

Beginning with the linear equations of motion [Eqs. (10a) and (10b)], we cross-differentiate to eliminate pressure and obtain a single partial differential equation to describe the cross-shore velocity component:

$$
S_{t} \frac{\partial^{2} \tilde{v}}{\partial \tilde{t} \partial \tilde{z}}+C_{b} \frac{\partial \tilde{u}}{\partial \tilde{z}}=\frac{\sin (2 \pi \tilde{t})}{2 \pi \tilde{y}^{2}}+(1+\gamma \mathcal{G}) \frac{\partial^{3} \tilde{v}}{\partial \tilde{z}^{3}}
$$

Here, the alongshore velocity $\tilde{u}(\tilde{z})$ is assumed to be given from an uncoupled alongshore momentum balance or from observations. We will seek a perturbation series solution in terms of the linear superposition

$$
\tilde{\boldsymbol{v}}=\tilde{\boldsymbol{v}}^{(0)}+S_{t} \tilde{\boldsymbol{v}}^{(1)}+\ldots,
$$

where $S_{t} \ll 1$ and we assume that all the perturbation parameters are of the same order, $S_{t} \sim \mathcal{W}_{b} \sim C_{b} \sim \gamma$. The solution in Eq. (A2) can alternately be written in terms of any of the other perturbation parameters. Perturbations that do not scale with the others would appear at higher orders. Substituting Eq. (A2) in Eq. (A1) and examining each order separately, the zeroth-order balance in Eq. (A1) is given by

$$
\frac{\partial^{3} \tilde{\boldsymbol{v}}^{(0)}}{\partial \tilde{z}^{3}}+\frac{\sin (2 \pi \tilde{t})}{2 \pi \tilde{y}^{2}}=0 .
$$

The no-slip condition on the bottom [Eq. (13a)] and null net transport [Eq. (13c)] must be satisfied by the velocity at all orders. The free surface boundary condition [Eq. (13b)] appears at first order and thus requires that the zeroth-order velocity satisfy zero stress. The solution for Eq. (A3) is thus

$$
\tilde{\boldsymbol{v}}^{(0)}=-\frac{\tilde{y}}{96 \pi}\left\{8\left(\frac{\tilde{z}}{\tilde{y}}\right)^{3}-9\left(\frac{\tilde{z}}{\tilde{y}}\right)^{2}+1\right\} \sin (2 \pi \tilde{t}),
$$

resulting from a balance between a cross-shore baroclinic gradient and vertical diffusion (Farrow and Patterson 1993). The effects of perturbations associated with Coriolis, wind stress, and time-variable turbulent mixing that alter the baseline solution appear then at $O\left(S_{t}\right)$. Collecting terms of $O\left(S_{t}\right)$, Eqs. (10a) and (10b) give

$$
\frac{\partial^{3} \tilde{\boldsymbol{v}}^{(1)}}{\partial \tilde{z}^{3}}=\frac{\partial^{2} \tilde{\boldsymbol{v}}^{(0)}}{\partial \tilde{t} \partial \tilde{z}}+\frac{C_{b}}{S_{t}} \frac{\partial \tilde{u}}{\partial \tilde{z}}-\frac{\gamma}{S_{t}} \mathcal{G} \frac{\partial^{3} \tilde{\boldsymbol{v}}^{(0)}}{\partial \tilde{z}^{3}},
$$

where $\tilde{\boldsymbol{v}}^{(1)}$ is again subject to the boundary conditions in Eq. (13).

The solution at $O\left(S_{t}\right)$ is obtained using the zerothorder solution, integrating three times with respect to $\tilde{z}$ :

$$
\begin{aligned}
\tilde{\boldsymbol{v}}^{(1)}= & \int_{\tilde{z}}^{0} \int_{\tilde{z}}^{0}\left(\frac{\partial \tilde{\boldsymbol{v}}^{(0)}}{\partial \tilde{t}}\right) d \tilde{z} d \tilde{z}+\frac{C_{b}}{S_{t}} \int_{\tilde{z}}^{0} \int_{\tilde{z}}^{0} \tilde{u} d \tilde{z} d \tilde{z}-\frac{\gamma}{S_{t}} \mathcal{G} \tilde{\boldsymbol{v}}^{(0)} \\
& +C_{1} \frac{\tilde{z}^{2}}{2}+C_{2} \tilde{z}+C_{3} .
\end{aligned}
$$

The functions $C_{1}, C_{2}$, and $C_{3}$ are constants respect to $\tilde{z}$ and are obtained from the boundary conditions.

To evaluate the surface wind stress boundary condition in Eq. (13b), we first differentiate Eq. (A6), to obtain $\frac{\partial \tilde{\boldsymbol{v}}^{(1)}}{\partial \tilde{z}}=\frac{\partial}{\partial \tilde{t}} \int_{\tilde{z}}^{0} \tilde{\boldsymbol{v}}^{(0)} d \tilde{z}+\frac{C_{b}}{S_{t}} \int_{\tilde{z}}^{0} \tilde{u} d \tilde{z}-\frac{\gamma}{S_{t}} \mathcal{G} \frac{\partial \tilde{\boldsymbol{v}}^{(0)}}{\partial \tilde{z}}+C_{1} \tilde{z}+C_{2}$.

Evaluating at $\tilde{z}=0$,

$$
\left.\frac{\partial \tilde{v}^{(1)}}{\partial \tilde{z}}\right|_{\tilde{z}=0}=C_{2}=\frac{\mathcal{W}_{b}}{S_{t}} \tilde{F}_{w}(\tilde{t})
$$

The no-slip boundary condition in Eq. (13a) applied to Eq. (A6) yields one equation for $C_{1}$ and $C_{3}$ :

$$
\begin{aligned}
C_{1} \frac{\tilde{y}^{2}}{2}+C_{3}= & -\frac{\mathcal{W}_{b}}{S_{t}} \tilde{y} \tilde{F}_{w}(\tilde{t})-\frac{\partial}{\partial \tilde{t}} \int_{\tilde{y}}^{0} \int_{\tilde{z}}^{0} \tilde{v}^{(0)} d \tilde{z} d \tilde{z} \\
& -\frac{C_{b}}{S_{t}} \int_{\tilde{y}}^{0} \int_{z}^{0} \tilde{u} d \tilde{z} d \tilde{z} .
\end{aligned}
$$

The second equation for $C_{1}$ and $C_{3}$ is obtained from the crossshore net flux condition in Eq. (13c), along with Eq. (A8):

$$
\begin{aligned}
\int_{\tilde{y}}^{0} \tilde{\boldsymbol{v}}^{(1)} d \tilde{z}= & \frac{\partial}{\partial \tilde{t}} \int_{\tilde{y}}^{0} \int_{\tilde{z}}^{0} \int_{\tilde{z}}^{0} \tilde{\boldsymbol{v}}^{(0)} d \tilde{z} d \tilde{z} d \tilde{z} \\
& +\frac{C_{b}}{S_{t}} \int_{\tilde{y}}^{0} \int_{\tilde{z}}^{0} \int_{\tilde{z}}^{0} \tilde{u} d \tilde{z} d \tilde{z} d \tilde{z}+C_{1} \frac{\tilde{y}^{3}}{6} \\
& +\frac{\mathcal{W}_{b}}{S_{t}} \frac{\tilde{y}^{2}}{2} \tilde{F}_{w}(\tilde{t})+C_{3} \tilde{y}=0 .
\end{aligned}
$$

Solving Eqs. (A9) and (A10) for $C_{1}$ and $C_{3}$ yields the following: 


$$
\begin{aligned}
C_{1}= & -\frac{3}{2} \frac{\mathcal{W}_{b}}{S_{t}} \frac{\tilde{F}_{w}(\tilde{t})}{\tilde{y}}-3 \frac{C_{b}}{S_{t}}\left\{I_{c, 1}(\tilde{u})-I_{c, 2}(\tilde{u})\right\} \\
& -3\left\{I_{u, 1}\left(\tilde{v}^{(0)}\right)-I_{u, 2}\left(\tilde{v}^{(0)}\right)\right\}, \quad \text { and } \\
C_{3}= & -\frac{1}{4} \frac{\mathcal{W}_{b}}{S_{t}} \tilde{y} \tilde{F}_{w}(\tilde{t})+\frac{\tilde{y}^{2}}{2} \frac{C_{b}}{S_{t}}\left\{I_{c, 1}(\tilde{u})-3 I_{c, 2}(\tilde{u})\right\} \\
& +\frac{\tilde{y}^{2}}{2}\left\{I_{u, 1}\left(\tilde{v}^{(0)}\right)-3 I_{u, 2}\left(\tilde{v}^{(0)}\right)\right\},
\end{aligned}
$$

$$
\begin{aligned}
& I_{c, 2}(\tilde{u})=\tilde{y}^{-3} \int_{\tilde{y}}^{0} \int_{\tilde{z}}^{0} \int_{\tilde{z}}^{0} \tilde{u} d \tilde{z} d \tilde{z} d \tilde{z}, \\
& I_{u, 1}(\tilde{u})=\tilde{y}^{-2} \frac{\partial}{\partial \tilde{t}} \int_{\tilde{y}}^{0} \int_{\tilde{z}}^{0} \tilde{v}^{(0)} d \tilde{z} d \tilde{z}
\end{aligned}
$$

and

where

$$
I_{c, 1}(\tilde{u})=\tilde{y}^{-2} \int_{\tilde{y}}^{0} \int_{\tilde{z}}^{0} \tilde{u} d \tilde{z} d \tilde{z}
$$

The solution for the cross-shore velocity at $O\left(S_{t}\right)$ can be written compactly as

$$
\begin{aligned}
S_{t} \tilde{\boldsymbol{v}}^{(1)}= & S_{t}\left\{\frac{\partial}{\partial \tilde{t}} \int_{\tilde{z}}^{0} \int_{\tilde{z}}^{0} \tilde{\boldsymbol{v}}^{(0)} d \tilde{z} d \tilde{z}-\frac{\tilde{y}^{2}}{2} I_{u, 1}\left(3 \frac{\tilde{z}^{2}}{\tilde{y}^{2}}-1\right)+\frac{3 \tilde{y}^{2}}{2} I_{u, 2}\left(\frac{\tilde{z}^{2}}{\tilde{y}^{2}}-1\right)\right\} \\
& +C_{b}\left\{\int_{\tilde{z}}^{0} \int_{z}^{0} \tilde{u} d \tilde{z} d \tilde{z}-\frac{\tilde{y}^{2}}{2} I_{c, 1}\left(3 \frac{\tilde{z}^{2}}{\tilde{y}^{2}}-1\right)+\frac{3 \tilde{y}^{2}}{2} I_{c, 2}\left(\frac{\tilde{z}^{2}}{\tilde{y}^{2}}-1\right)\right\}+\mathcal{W}_{b}\left\{\frac{\tilde{y}}{4}\left(3 \frac{\tilde{z}^{2}}{\tilde{y}^{2}}-4 \frac{\tilde{z}}{\tilde{y}}+1\right) \tilde{F}_{w}\right\}+\gamma\left\{-\mathcal{G} \tilde{v}^{(0)}\right\} .
\end{aligned}
$$

The terms in braces on the right-hand side of Eq. (A13) can be interpreted, respectively, as corresponding to 1) the unsteady velocity perturbation, 2) the Coriolis-driven velocity perturbation, 3 ) wind-driven velocity component, and 4) the velocity perturbation resulting from the time-varying eddy viscosity. Using the Stokes boundary layer solution given in Eq. (20) for the alongshore velocity and rewriting the $O\left(S_{t}\right)$ velocity component as

$$
S_{t} \tilde{v}^{(1)}=S_{t} \tilde{v}_{u}+\mathcal{W}_{b} \tilde{v}_{w}+C_{b} \tilde{v}_{c}+\gamma \tilde{v}_{t},
$$

we can evaluate the integrals in Eq. (A12) and solve for the perturbation components in Eq. (A13) as follows:

$$
\begin{aligned}
\tilde{v}_{u}= & -\frac{\tilde{y}^{3}}{8}\left\{\frac{1}{15}\left(\frac{\tilde{z}}{\tilde{y}}\right)^{5}-\frac{1}{8}\left(\frac{\tilde{z}}{\tilde{y}}\right)^{4}+\frac{1}{15}\left(\frac{\tilde{z}}{\tilde{y}}\right)^{2}\right. \\
& \left.-\frac{1}{120}\right\} \cos (2 \pi \tilde{t}), \\
\tilde{v}_{w}= & \frac{\tilde{y}}{4}\left\{3\left(\frac{\tilde{z}}{\tilde{y}}\right)^{2}-4\left(\frac{\tilde{z}}{\tilde{y}}\right)+1\right\} \\
& \times\left\{A+B \sin \left(2 \pi \tilde{t}+\phi_{(w)}\right)\right\}, \\
\tilde{v}_{c}= & \Re\left\{\hat{C} \tilde{v}_{c} e^{\left.i\left[2 \pi\left(\omega_{\mathrm{M}_{2}} / \omega_{T}\right) \tilde{t}-\phi_{\mathrm{M}_{2}}\right]\right\}, \quad \text { and }}\right.
\end{aligned}
$$

$$
\tilde{v}_{t}=-\mathcal{G} \tilde{v}_{\nu}
$$

In Eq. (A15c),

$$
\begin{aligned}
\hat{v}_{c}= & i \frac{\tilde{\delta}_{\nu}^{2}}{4}\left\{2 \frac{\cosh \left\{(1+i) \tilde{z} / \tilde{\delta}_{\nu}\right\}}{\cosh \left\{(1+i) \tilde{y} / \tilde{\delta}_{\nu}\right\}}+1-3\left(\frac{\tilde{z}}{\tilde{y}}\right)^{2}\right\} \\
& -\frac{3}{8}(1+i) \frac{\tilde{\delta}_{\nu}^{3}}{\tilde{y}}\left\{1-\left(\frac{\tilde{z}}{\tilde{y}}\right)^{2}\right\} \\
& \times \tanh \left\{(1+i) \frac{\tilde{y}}{\tilde{\delta}_{\nu}}\right\}
\end{aligned}
$$

and

$$
\hat{C}=-\left\{\tilde{y}-(1-i) \frac{\tilde{\delta}_{v}}{2} \tanh \left\{(1+i) \frac{\tilde{y}}{\tilde{\delta}_{\nu}}\right\}\right\}^{-1} .
$$

The fact that the unsteady term appears at $O\left(S_{t}\right)$ in Eq. (10a) means that the leading-order flow is limited to steady or harmonic solutions and cannot address transients associated with arbitrary initial conditions. Time dependence in the solutions in Eq. (A15) is then reflective of harmonic components in the forcing functions for heat flux, wind, and alongshore flow.

We highlight that the solution in Eq. (A2) is controlled by the unsteady buoyancy/diffusive regime that leads to the baseline flow $\tilde{\boldsymbol{v}}^{(0)}$ in Eq. (A4). A spatial limit on the validity of the solution can be derived 
considering the behavior of the various components of solution versus cross-shore distance $\tilde{y}$. In particular, it is noteworthy that the baseline cross-shore velocity $\tilde{\boldsymbol{v}}^{(0)}$ increases linearly with $\tilde{y}$, while the unsteady velocity component $\tilde{v}_{u}$ increases as $\tilde{y}^{3}$. Applying this with Eq. (A14) in Eq. (A2), it is apparent that the perturbation assumption of $\tilde{\boldsymbol{v}}^{(1)} \ll \tilde{\boldsymbol{v}}^{(0)}$ will be violated when $\tilde{y} \sim S_{t}^{-1 / 2}$, or equivalently, when the depth is of order $\sqrt{v_{e} \mathcal{I}_{s}}$, where the local acceleration must be considered at leading order.

\section{APPENDIX B}

\section{Free-Surface Stokes Boundary Layer}

The Stokes boundary layer flow with free surface in Eq. (20) is derived by considering a linear unsteady/ diffusive momentum balance forced by a periodic alongshore pressure gradient,

$$
\frac{\partial \tilde{u}}{\partial \tilde{t}}=-\tilde{P}_{x}+\frac{v_{e}}{\omega_{u} D_{0}^{2}} \frac{\partial^{2} \tilde{u}}{\partial \tilde{z}^{2}},
$$

subject to no-slip, $\tilde{u}(\tilde{z}=\tilde{y})=0$, and free-slip, $\partial_{\tilde{z}} \tilde{u}(\tilde{z}=$ $0)=0$, boundary conditions on the bottom and the free surface, respectively. The initial condition, $\tilde{u}\left(\tilde{t}=\tilde{t}_{0}\right)=\tilde{U}_{0}$, depends on the flow phase. The pressure gradient is simply modeled as $\tilde{P}_{x}=C \exp \left(i \omega_{u} \tilde{t}-i 2 \pi \phi_{u}\right)$, where $\omega_{u}$ and $\phi_{u}$ are the forcing frequency and its phase, respectively. The function $C$ is a constant with respect to $\tilde{z}$ and is obtained from the following condition:

$$
\int_{\tilde{y}}^{0} \hat{u}(\tilde{z}) d \tilde{z}=1 .
$$

The PDE in Eq. (B1) is solved by looking for periodic solutions of the following form:

$$
\tilde{u}=\hat{u}(\tilde{z}) \exp \left(i 2 \pi \frac{\omega_{u}}{\omega_{s}} \tilde{t}-i \phi_{u}\right) .
$$

The spatial component $\hat{u}(\tilde{z})$ is obtained using Eq. (B3) in Eq. (B1). Integration of the resulting second-order ODE yields the spatial component in Eq. (20).

\section{REFERENCES}

Alford, M., M. Gregg, and M. Merrifield, 2006: Structure, propagation and mixing of energetic baroclinic tides in Mamala Bay, Oahu, Hawaii. J. Phys. Oceanogr., 36, 997-1018, https:// doi.org/10.1175/JPO2877.1.

Biton, E., J. Silverman, and H. Gildor, 2008: Observations and modeling of a pulsating density current. Geophys. Res. Lett., 35, L14603, https://doi.org/10.1029/2008gl034123.
Brink, K. H., 2016: Cross-shelf exchange. Annu. Rev. Mar. Sci., 8, 59-78, https://doi.org/10.1146/annurev-marine-010814-015717.

Cormack, D. E., G. P. Stone, and L. G. Leal, 1975: The effect of upper surface conditions on convection in a shallow cavity with differentially heated end-walls. Int. J. Heat Mass Transfer, 18, 635-648, https://doi.org/10.1016/0017-9310(75) 90275-6.

Davis, K. A., and S. G. Monismith, 2011: The modification of bottom boundary layer turbulence and mixing by internal waves shoaling on a barrier reef. J. Phys. Oceanogr., 41, 22232241, https://doi.org/10.1175/2011JPO4344.1.

_ J. J. Leichter, J. L. Hench, and S. G. Monismith, 2008: Effects of western boundary current dynamics on the internal wave field of the southeast Florida shelf. J. Geophys. Res., 113, C09010, https://doi.org/10.1029/2007JC004699.

Farrow, D. E., 2002: Coriolis effects and the thermal bar. J. Geophys. Res., 107, 3033, https://doi.org/10.1029/2000JC000727.

_- 2013: Periodically driven circulation near the shore of a lake. Environ. Fluid Mech., 13, 243-255, https://doi.org/10.1007/ s10652-012-9261-4.

— , and J. C. Patterson, 1993: On the response of a reservoir sidearm to diurnal heating and cooling. J. Fluid Mech., 246, 143-161, https://doi.org/10.1017/S0022112093000072.

— , and —, 1994: The daytime circulation and temperature structure in a reservoir sidearm. Int. J. Heat Mass Transfer, 37, 1957-1968, https://doi.org/10.1016/0017-9310(94)90335-2.

Fischer, H. B., J. E. List, and C. R. Koh, 1979: Mixing in Inland and Coastal Waters. Academic Press, 302 pp.

Grant, W. D., and O. S. Madsen, 1979: Combined wave and current interaction with a rough bottom. J. Geophys. Res., 84, 17971808, https://doi.org/10.1029/JC084iC04p01797.

Hasselmann, K., 1970: Wave-driven inertial oscillations. Geophys. Astrophys. Fluid Dyn., 1, 463-502, https://doi.org/10.1080/ 03091927009365783.

Herdman, L. M. M., J. L. Hench, and S. G. Monismith, 2015: Heat balance and thermally driven lagoon-ocean exchanges on a tropical reef system (Moorea, French Polynesia). J. Geophys. Res. Oceans, 120, 1233-1252, https://doi.org/ 10.1002/2014JC010145.

Jones, N. L., R. J. Lowe, G. Pawlak, D. A. Fong, and S. G. Monismith, 2008: Plume dispersion on a fringing coral reef system. Limnol. Oceanogr., 53, 2273-2286, https://doi.org/ 10.4319/lo.2008.53.5_part_2.2273.

Kumar, N., and F. Feddersen, 2017: The effect of Stokes drift and transient rip currents on the inner shelf. Part I: No stratification. J. Phys. Oceanogr., 47, 227-241, https://doi.org/10.1175/ JPO-D-16-0076.1.

Leichter, J. J., S. R. Wing, S. L. Miller, and M. W. Denny, 1996: Pulsed delivery of subthermocline water to Conch Reef (Florida Keys) by internal tidal bores. Limnol. Oceanogr., 41, 1490-1501, https://doi.org/10.4319/lo.1996.41.7.1490.

—, H. L. Stewart, and S. L. Miller, 2003: Episodic nutrient transport to Florida coral reefs. Limnol. Oceanogr., 48, 1394 1407, https://doi.org/10.4319/lo.2003.48.4.1394.

Lentz, S. J., and M. Fewings, 2012: The wind- and wave-driven inner-shelf circulation. Annu. Rev. Mar. Sci., 4, 317-343, https://doi.org/10.1146/annurev-marine-120709-142745.

,,-- P. Howd, J. Fredericks, and K. Hathaway, 2008: Observations and a model of undertow over the inner continental shelf. J. Phys. Oceanogr., 38, 2341-2357, https://doi.org/ 10.1175/2008JPO3986.1.

—, K. A. Davis, J. H. Churchill, and T. M. DeCarlo, 2017: Coral reef drag coefficients-Water depth dependence. J. Phys. 
Oceanogr., 47, 1061-1075, https://doi.org/10.1175/JPO-D-160248.1.

Lin, Y.-T., 2015: Wind effect on diurnal thermally driven flow in vegetated nearshore of a lake. Environ. Fluid Mech., 15, 161-178, https://doi.org/10.1007/s10652-014-9368-x.

Lowe, R. J., and J. L. Falter, 2015: Oceanic forcing of coral reefs. Annu. Rev. Mar. Sci., 7, 43-66, https://doi.org/10.1146/ annurev-marine-010814-015834.

,-- S. G. Monismith, and M. J. Atkinson, 2009: Wave-driven circulation of a coastal reef-lagoon system. J. Phys. Oceanogr., 39, 873-893, https://doi.org/10.1175/2008JPO3958.1.

Mao, Y., C. Lei, and J. C. Patterson, 2009: Unsteady natural convection in a triangular enclosure induced by absorption of radiation-A revisit by improved scaling analysis. J. Fluid Mech., 622, 75-102, https://doi.org/10.1017/S0022112008005077.

Molina, L., G. Pawlak, J. R. Wells, S. G. Monismith, and M. A. Merrifield, 2014: Diurnal cross-shore thermal exchange on a tropical forereef. J. Geophys. Res. Oceans, 119, 6101-6120, https://doi.org/10.1002/2013JC009621.

Monismith, S. G., 2007: Hydrodynamics of coral reefs. Annu. Rev. Fluid Mech., 39, 37-55, https://doi.org/10.1146/ annurev.fluid.38.050304.092125.
_ J. Imberger, and M. L. Morison, 1990: Convective motions in the sidearm of a small reservoir. Limnol. Oceanogr., 35, 16761702, https://doi.org/10.4319/lo.1990.35.8.1676.

_ - A. Genin, M. A. Reidenbach, G. Yahel, and J. R. Koseff, 2006: Thermally driven exchange between a coral reef and the adjoining ocean. J. Phys. Oceanogr., 36, 1332-1347, https://doi.org/ 10.1175/JPO2916.1.

Pawlak, G., and Coauthors, 2009: Development, deployment, and operation of Kilo Nalu nearshore cabled observatory. OCEANS 2009, Bremen, Germany, IEEE, 10914940, https:// doi.org/10.1109/OCEANSE.2009.5278149.

Sevadjian, J., M. McManus, and G. Pawlak, 2010: Effects of physical structure and processes on thin zooplankton layers in Mamala Bay, Hawai'i. Mar. Ecol. Prog. Ser., 409, 95-106, https://doi.org/10.3354/meps08614.

Smith, K. A., G. Rocheleau, M. A. Merrifield, S. Jaramillo, and G. Pawlak, 2016: Temperature variability caused by internal tides in the coral reef ecosystem of Hanauma Bay, Hawai'i. Cont. Shelf Res., 116, 1-12, https://doi.org/10.1016/ j.csr.2016.01.004.

Willmott, C. J., 1981: On the validation of models. Phys. Geogr., 2 , 184-194, https://doi.org/10.1080/02723646.1981.10642213. 